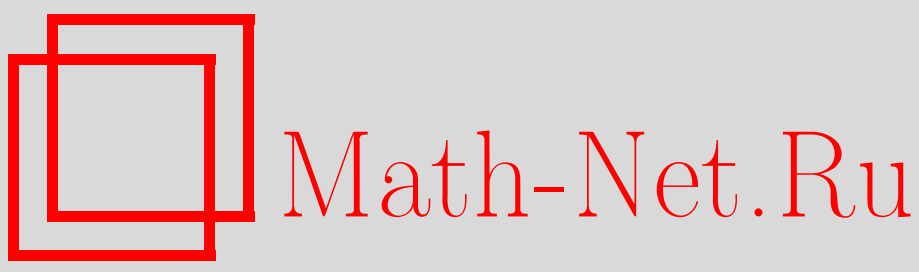

А. Ю. Савин, Б. Ю. Стернин, Эллиптические операторы в четных подпространствах, Матем. сб., 1999, том 190, номер 8, 125-160

DOI: https://doi.org/10.4213/sm423

Использование Общероссийского математического портала Math-Net.Ru подразумевает, что вы прочитали и согласны с пользовательским соглашением

http://www . mathnet.ru/rus/agreement

Параметры загрузки:

IP: 54.162 .27 .143

26 апреля 2023 г., $16: 57: 23$ 


\title{
Эллиптические операторы в четных подпространствах
}

\begin{abstract}
Строится эллиптическая теория для операторов, действующих в подпространствах, определяемых четньми псевдодифференциальньми проекторами. Доказана формула индекса для компактных многообразий без края и для общих краевых задач. Найдена связь с теорией $\eta$-инвариантов Гилки.

Библиографоия: 17 названий.
\end{abstract}

\section{Введение}

Как следует из заглавия, в работе изучаются эллиптические операторы в подпространствах, определяемых проекторами, более точно, четными псевдодифференциальньми проекторами. Такие проекторы возникают уже в классических краевых задачах. В самом деле, при сведении эллиптической краевой задачи на край соответствующий оператор, как показьвает анализ, действует в подпространствах, определяемых (псевдодифференциальньми) проекторами. Более того, проекторы оказываются чрезвычайно полезными для построения теории Фредгольма для эллиптических операторов, свободных от известного условия АтьиБотта [1]. Как показано в статье [2], такая теория может быть построена именно в подпространствах пространств Соболева, определяемых псевдодифференциальньми проекторами. Это в действительности результаты, восходящие к классическим пространствам Харди, в которых (и только в них) может быть построена корректная (фредгольмова) теория для оператора Коши-Римана. Важным классом проекторов является класс четных проекторов. Точные определения будут даны позднее, а сейчас мы только укажем, что в подпространствах, определяемых такими проекторами, удается доказать не только теорему конечности, но и предъявить соответствующую формулу индекса.

Пожалуй, самым главным и принципиальным отличием эллиптической теории в подпространствах, определяемых проекторами, от аналогичной теории в соболевских пространствах является то, что хотя понятие эллиптичности, как и в классическом случае, определяется главными символами основного оператора и проекторов, индекс эллиптического оператора в подпространствах уже не определяется не только главным символом проекторов, но и их полными символами. Это приводит к необходимости дать некоторую численную характеристику псевдодифференциальных проекторов или, что то же самое, подпространств, определяемых

Работа выполнена при поддержке Российского фонда фундаментальных исследований (гранты № № 97-01-00703, 97-02-16722а), Международного научного фонда (грант № а99-1763) и Arbeitsgruppe Partielle Differentialgleichungen und Komplexe Analysis, Institut für Mathematik, Universität Potsdam.

(C) А.Ю. САвин, Б. Ю. Стернин 1999 
ими. Такую численную характеристику, играющую в определенном смысле роль размерности проектора (и совпадаюшую в конечномерном случае с рангом проектора), удается предъявить по крайней мере в классе четных проекторов. Это понятие, несомненно, является фундаментальньм в данной теории. В этих терминах мы получаем формулу индекса в ситуации компактных многообразий без края и для общих краевых задач, а также устанавливаем связь введенного понятия "размерности" с $\eta$-инвариантом Атьи-Патоди-Зингера [3].

В $\S 1$ на нечетномерных компактных многообразиях $M$ без края рассматриваются подпространства, которые определяются как образы псевдодифференциальных проекторов. $^{1}$ Более точно, предполагается, что проекторы являются четнылми в том смысле, что их главный символ является четной функцией по импульсньм (кокасательным) переменным.

Оказывается, что в классе таких подпространств существует однозначно определенный, с точностью до нормировки, аналог понятия размерности конечномерных векторных пространств (т.е. гомотопически инвариантный аддитивный функционал). При этом нормировка задает размерность пространств сечений расслоений на многообразии $M$.

Четные проекторы обладают следуюшим свойством: группа классов стабильно гомотопически эквивалентных четных проекторов рационально порождена элементами, которые отличаются от проекторов на пространства сечений расслоений на многообразии $M$ на конечномерный оператор. Это утверждение в действительности является следствием того, что препятствие к стабильной гомотопии проекторов (по модулю конечномерных операторов) лежит в группе $K\left(P^{*} M\right) / K(M)$ (здесь $P^{*} M-$ проективизация расслоения кокасательных сфер), которая целиком состоит из кручения.

В $§ 2$ изучаются эллиптические операторы, действующие в подпространствах, определяемых псевдодифференциальными проекторами

$$
D: H_{1} \rightarrow H_{2}, \quad H_{1,2}=\operatorname{Im} P_{1,2} \text {. }
$$

Именно, показано, что индекс эллиптических операторов рассматриваемого вида представляется в виде суммы двух слагаемых, одно из которых определяется только главньм символом оператора $D$ (и является гомотопическим инвариантом главного символа задачи), а второе определяется лишш подпространствами, в которых действует оператор $D$, и является гомотопическим инвариантом подпространств.

Отметим, что разложение такого вида отсутствует в классе всех эллиптических операторов. Можно показать, что здесь, так же как и в теории спектральных краевых задач (см. [4], [5]), возникает препятствие к такому разбиению, которое тесно связано со спектральными потоками периодических семейств операторов (см. $\S 1)$. Вот почему для получения формулы индекса необходимо брать более узкие классы операторов.

Далее в $\S \S 3-5$ дается приложение развитой теории к краевым задачам. Именно, в $\S 3$ рассматриваются общие краевые задачи [2]

$$
\left\{\begin{array}{l}
D u=f, \\
B u=g \in \operatorname{Im} P,
\end{array}\right.
$$

\footnotetext{
${ }^{1}$ Отметим, что все элементы приводимых ниже конструкций определяются подпространствами и не зависят от выбора проекторов на эти подпространства.
} 
где $D$ - эллиптический дифференциальньй оператор на многообразии с краем, $B$ некоторый граничный оператор, а $P$ - псевдодифференциальный проектор на крае $\partial M$ гладкого многообразия $M$. Этот класс краевых задач, с одной стороны, содержит все классические краевые задачи с условием Лопатинского (см., например, [6]), с другой стороны, для любого эллиптического оператора $D$ в этом классе существует фредгольмова краевая задача. Здесь даются все необходимые определения, приводятся примеры.

Параграфы 4, 5 посвящены собственно проблеме вычисления индекса общих краевых задач. Так, в $§ 4$ задача редуцируется к некоторой спектральной задаче [7], которая в воротниковой окрестности края с нормальной координатой $t$ имеет вид

$$
\left\{\begin{array}{l}
\left(\frac{\partial}{\partial t}+A(t)\right) u=f \\
\left.P u\right|_{\partial M}=g \in \operatorname{Im} P
\end{array}\right.
$$

где однородный главный символ псевдодифференциального оператора $A(0)$ на расслоении кокасательных сфер к краю равен $\sigma(A(0))=2 \sigma(P)-1$. Редукция здесь понимается в смысле совпадения индексов соответствующих фредгольмовых операторов.

В $\S 5$ рассматривается класс четных краевых задач на четномерном многообразии $M$, т.е. краевых задач вида (1) с четньм проектором $P$. При таком условии удается получить формулу индекса для спектральных краевых задач (2)

$$
\operatorname{ind}(D, P)=\frac{1}{2} \operatorname{ind}_{t}(\sigma(\widetilde{D}))-d(P)
$$

где через $\sigma(\widetilde{D})$ обозначен эллиптический символ на дубле многообразия $M$, получаемый (непрерьвной) склейкой символов $\sigma(D)(\xi)$ и $\sigma(D)(-\xi)$, а через $d(P)$ обозначен упомянутый выше функционал размерности при нулевой нормировке. Заметим, что в силу редукции из первой части работы формула (3) решает проблему индекса общих краевых задач (1) для четных проекторов $P$.

Третье приложение введенного понятия $d$-размерности связано с $\eta$-инвариантом Атьи-Патоди-Зингера [3]. Именно, инвариант $d$ четного псевдодифференциального проектора оказывается теснейшим образом связанным с теорией $\eta$-инвариантов самосопряженных эллиптических операторов четного порядка на нечетномерных многообразиях. Более точно, $\eta$-инвариант допустимого оператора [8] (см. также §6) в этом случае равен указанной выше "размерности” неотрицательного подпространства оператора для нулевой нормировки. Поэтому дробные части "размерности" и $\eta$-инварианта определяют один и тот же гомоморфизм

$$
K\left(P^{*} M\right) / K(M) \rightarrow \mathbb{Z}\left[\frac{1}{2}\right] \bmod \mathbb{Z} .
$$

В силу такого отождествления формула индекса (3) может быть интерпретирована как аналог формулы Атьи-Патоди-Зингера [3]. В качестве следствия формулы индекса в подпространствах получаем (см. следствие 1) топологическое выражение для дробной части $\eta$-инварианта на подгрупше

$$
\operatorname{ker}\left\{\pi^{*}: K\left(P^{*} M\right) / K(M) \rightarrow K\left(S^{*} M\right) / K(M)\right\}, \quad \pi: S^{*} M \rightarrow P^{*} M,
$$


а формула индекса четных краевых задач показывает инвариантность относительно кобордизма дробной части удвоенного $\eta$-инварианта (см. следствия 2,3 ). Оказывается, что в обоих случаях $\eta$-инвариант имеет двойку в качестве знаменателя или является целочисленным. Тем не менее, проблема нетривиальности этой дробной части остается открытой.

В последнем параграфе рассмотрены примеры.

Авторы признательны профессору А. С. Мишенко за ряд ценных замечаний, высказанных им при докладе этой работы на его семинаре в Московском Университете (осень 1998 г.). Он также посоветовал включить в данный текст приложения, касающиеся краевых задач, отчего статья, безусловно, выиграла. Мы также сердечно признательны В.Е. Назайкинскому за конструктивные предложения, позволившие улучшить первоначальный вариант работы. Наконец, мы благодарны профессору П. Гилки, дискуссия с которым по поводу данной работы была для нас чрезвычайно полезной.

\section{§1. Четные псевдодифференциальные проекторы}

Будем рассматривать множество $\operatorname{Proj}(M)$ псевдодифференциальных проекторов нулевого порядка, действующих в пространствах гладких сечений векторных расслоений на замкнутом многообразии $M$. В этом множестве невозможно сравнивать ранги (размерности образов) проекторов так, как это можно делать в случае конечномерных пространств. Более точно, на пространстве псевдодифференциальных проекторов не существует гомотопически инвариантного (в смысле операторной $L^{2}$-нормы) функционала

$$
d: \operatorname{Proj}(M) \rightarrow \mathbb{Z} \quad \text { (и даже } \mathbb{R} \text { или } \mathbb{C}),
$$

которьй бы удовлетворял (слабому) свойству аддитивности

$$
d\left(P \oplus P^{\prime}\right)=d(P)+\operatorname{dim} \operatorname{Im} P^{\prime}
$$

где $P$ - произвольный проектор, а $P^{\prime}$ - конечномерный проектор. В самом деле, предположив противное, рассмотрим произвольное периодическое семейство $A_{t}$ самосопряженных эллиптических операторов с ненулевым спектральньм потоком. Такие семейства сушествуют (см., например, [4]).

Обозначим соответствуюшее семейство проекторов на неотрицательные спектральные подпространства операторов $A_{t}$ через $P_{t}$. Тогда из определения спектрального потока sf $A_{t}$ как алгебраического числа собственных значений операторов $A_{t}$, которые проходят через нуль при изменении параметра $t$, получаем

$$
\operatorname{sf} A_{t}=d\left(P_{1}\right)-d\left(P_{0}\right) \neq 0
$$

Но семейство $A_{t}$ является периодическим. Следовательно, $P_{0}=P_{1}$ и поэтому $d\left(P_{1}\right)=d\left(P_{0}\right)$, что находится в противоречии с формулой $(4)$.

В параграфе рассматривается также класс так называемых четных проекторов, в котором аналогичные функционалы все-таки могут быть определены при усло-

вии, что многообразие $M$, над которым они действуют, является нечетномерным. 
ОПРЕДЕЛЕНИЕ 1. Псевдодифференциальный проектор

$$
P: C^{\infty}(M, E) \rightarrow C^{\infty}(M, E)
$$

называется четным (ср. [8]), если его однородный главный символ удовлетворяет соотношению

$$
\sigma(P)(x, \xi)=\sigma(P)(x,-\xi) \text { для всех }(x, \xi) \in S^{*} M
$$

Множество всех четных псевдодифференциальных проекторов нулевого порядка обозначим через $\operatorname{Even}(M)$. Пусть

$$
P^{*} M=S^{*} M / \mathbb{Z}_{2}
$$

- расслоение проективных пространств, полученное как факторпространство расслоения кокасательных сфер $S^{*} M$ по действию антиподальной инволюции $\xi \rightarrow-\xi$. Соответствуюшую проекцию обозначим через $\pi: S^{*} M \rightarrow P^{*} M$. Тогда условие симметрии (5) проектора $P$ означает в точности то, что однородный главный символ $\sigma(P)$ является поднятием эндоморфизма $\sigma^{\prime}(P)$ с проективных пространств $P^{*} M$ :

$$
\sigma(P)=\pi^{*} \sigma^{\prime}(P), \quad \sigma^{\prime}(P): \pi_{P}^{*} E \rightarrow \pi_{P}^{*} E
$$

где $\pi_{P}: P^{*} M \rightarrow M$ - естественная проекция. Заметим, что из равенства (6) следует, что четный проектор задает векторное расслоение над проективизацией $P^{*} M$

$$
\operatorname{Im} \sigma^{\prime}(P) \in \operatorname{Vect}\left(P^{*} M\right)
$$

Пусть $P_{1,2}$ - псевдодифференциальные проекторы

$$
P_{1,2}: C^{\infty}\left(M, E_{1,2}\right) \rightarrow C^{\infty}\left(M, E_{1,2}\right)
$$

Тогда прямая сумма $P_{1} \oplus P_{2}$ представляет собой проектор

$$
P_{1} \oplus P_{2}=\left(\begin{array}{cc}
P_{1} & 0 \\
0 & P_{2}
\end{array}\right): C^{\infty}\left(M, E_{1} \oplus E_{2}\right) \rightarrow C^{\infty}\left(M, E_{1} \oplus E_{2}\right)
$$

На множестве $\operatorname{Even}(M)$ четных проекторов рассмотрим следующее отношение стабильной гомотопической эквивалентности. 
ОПРеДЕЛЕНИЕ 2 . Будем назьвать проекторы $P_{1,2} \in \operatorname{Even}(M)$ әквивалентными и писать $P_{1} \approx P_{2}$, если для некоторого четного проектора $P: C^{\infty}(M, F) \rightarrow$ $C^{\infty}(M, F)$ сушествует гомотопия четных проекторов

$$
P_{1} \oplus 0 \oplus P \sim 0 \oplus P_{2} \oplus P
$$

как операторов, действующих в объемлюшем пространстве $C^{\infty}\left(M, E_{1} \oplus E_{2} \oplus F\right)$.

Рассмотрим группу Гротендика, порожденную полугруппой $\operatorname{Even}(M) / \approx$, состоящей из классов эквивалентных проекторов

$$
K\left(P_{\mathrm{ev}}(M)\right)=K(\operatorname{Even}(M) / \approx) .
$$

При этом образ проектора $P$ в группе $K\left(P_{\mathrm{ev}}(M)\right)$ будем обозначать через $[P]$. Ясно, что, сопоставляя четному псевдодифференциальному проектору образ его главного символа, получаем гомоморфизм абелевых групп

$$
q: K\left(P_{\mathrm{ev}}(M)\right) \rightarrow K\left(P^{*} M\right) .
$$

Упомянутые выше свойства размерности проекторов формализуем в виде следуюшего определения.

ОпРЕДЕЛЕНИЕ 3. Гомоморфизм групा

$$
d: K\left(P_{\mathrm{ev}}(M)\right) \rightarrow \mathbb{R}
$$

будем называть размерностью, если для любого конечномерного проектора $P$ имеет место нормировка

$$
d([P])=\operatorname{dim} \operatorname{Im} P .
$$

Следующая теорема описьвает все возможные размерности.

Теорема 1. Размерности четных подпространств

$$
d: K\left(P_{\mathrm{ev}}(M)\right) \rightarrow \mathbb{R}
$$

находятся во взаимно однозначном соответствии с гомоморфизмами нормировки

$$
\chi: K(M) \rightarrow \mathbb{R}
$$

При этом для целочисленньх нормировок х размерность д принимает рациональные значения, у которьх в знаменателе могут находиться лишь степени двойки:

$$
d: K\left(P_{\mathrm{ev}}(M)\right) \rightarrow \mathbb{Z}\left[\frac{1}{2}\right]
$$


ДокАЗАТЕЛьСтво. Рассмотрим последовательность

$$
0 \longrightarrow \mathbb{Z} \stackrel{i}{\longrightarrow} K\left(P_{\mathrm{ev}}(M)\right) \stackrel{q}{\longrightarrow} K\left(P^{*} M\right) \longrightarrow 0,
$$

где отображение $i$ индуцировано гомоморфизмом полугрупп

$$
\mathbb{Z}_{+} \rightarrow \operatorname{Even}(M) / \approx
$$

сопоставляющем неотрицательному числу $k$ конечномерньй проектор ранга $k$, действующий, например, в пространстве $C^{\infty}(M)$. Докажем, что последовательность (9) точна.

Проверим сначала, что гомоморфизм $q$ является эпиморфизмом. Действительно, произвольное векторное расслоение $\gamma \in \operatorname{Vect}\left(P^{*} M\right)$ можно реализовать как подрасслоение в некотором тривиальном расслоении

$$
\gamma \subset \mathbb{C}^{N} \in \operatorname{Vect}\left(P^{*} M\right) .
$$

Поднимая это вложение на кокасательные сферы, получаем

$$
\pi^{*} \gamma \subset \mathbb{C}^{N} \in \operatorname{Vect}\left(S^{*} M\right) .
$$

Очевидно, ортогональньй проектор $\sigma=\sigma(x, \xi)$ на подрасслоение $\pi^{*} \gamma$ является четным. Рассмотрим произвольный псевдодифференциальньй проектор $P$ с главным символом, равным $\sigma .^{2}$ Тогда для проектора $P$ по построению получаем

$$
q([P])=[\gamma] \in K\left(P^{*} M\right)
$$

что и доказывает точность последовательности в третьем члене.

Тривиальность сквозного отображения $q \circ i$ очевидна, поэтому проверим вложение $\operatorname{ker} q \subset \operatorname{Im} i$. Предположим, что

$$
q\left([P]-\left[1_{N}\right]\right)=0
$$

где $P$ - четный проектор в пространстве $C^{\infty}(M, E)$, а $1_{N}$ - единичный проектор в $C^{\infty}\left(M, \mathbb{C}^{N}\right)$ (это не ограничивает общности, так как произвольный элемент группы $K\left(P_{\text {ev }}(M)\right)$ представим в таком виде). Условие $(10)$ означает, что (быть может, после прибавления к паре $\left(P, 1_{N}\right)$ тривиальной пары $\left.\left(1_{N^{\prime}}, 1_{N^{\prime}}\right)\right)$ имеет место изоморфизм расслоений над $P^{*} M$

$$
\operatorname{Im} \sigma^{\prime}(P) \simeq \mathbb{C}^{N}
$$

${ }^{2}$ Следуя [9], такой проектор можно построить по формуле

$$
P=-\frac{1}{2 \pi i} \int_{|\lambda-1|=\varepsilon}(\Pi-\lambda I)^{-1} d \lambda
$$

где П - произвольньй псевдодифференциальный оператор нулевого порядка с главньм символом, равным $\sigma$, а число $\varepsilon, 0<\varepsilon<1$, выбрано таким образом, что на окружности $|\lambda-1|=\varepsilon$ нет собственных значений оператора П. 
Отсюда следует, что как подрасслоения в прямой сумме $\pi_{P}^{*} E \oplus \mathbb{C}^{N}$ они являются гомотопными. Рассмотрим произвольную соединяющую их гомотопию и обозначим соответствуюшее семейство проекторов через $\left\{\sigma_{t}^{\prime}\right\}_{t=0,1}$ :

$$
\begin{gathered}
\sigma_{0}^{\prime}=\sigma^{\prime}(P) \oplus 0, \quad \sigma_{1}^{\prime}=0 \oplus 1_{N}, \\
\operatorname{Im} \sigma_{t}^{\prime} \subset \pi_{P}^{*} E \oplus \mathbb{C}^{N} \in \operatorname{Vect}\left(P^{*} M\right) \text { для всех } t \in[0,1] .
\end{gathered}
$$

Поднятие этой гомотопии на расслоение сфер $S^{*} M$ обозначим через $\sigma_{t}$. Из утверждения 1 работы [10] следует, что сушествует (непрерывная) накрывающая гомотопия псевдодифференциальных проекторов $P_{t}$ :

$$
\sigma\left(P_{t}\right)=\sigma_{t}
$$

такая, что $P_{0}=P \oplus 0$, а $P_{1}$ отличается от $0 \oplus 1_{N}$ на компактный оператор. Поэтому в группе $K\left(P_{\mathrm{ev}}(M)\right)$ получаем равенство

$$
[P]-\left[1_{N}\right]=\left[P_{1}\right]-\left[0 \oplus 1_{N}\right]
$$

Напомним, что для проекторов $P, Q$ с компактной разностью относительным индексом называют индекс фредгольмова оператора $Q: \operatorname{Im} P \rightarrow \operatorname{Im} Q$

$$
\operatorname{ind}(P, Q) \stackrel{\text { def }}{=} \operatorname{ind}(Q: \operatorname{Im} P \rightarrow \operatorname{Im} Q)=-\operatorname{ind}(P: \operatorname{Im} Q \rightarrow \operatorname{Im} P) .
$$

Хорошо известно (см., например, [11]), что проекторы, отличающиеся на компактный оператор, гомотопны с точностью до проектора конечного ранга. Например, в случае положительного относительного индекса проекторов $\operatorname{ind}\left(P_{1}, 0 \oplus 1_{N}\right)=n \geqslant 0$ получаем гомотопию проекторов

$$
P_{1} \sim n \oplus 1_{N}
$$

где $n$ обозначает проектор размерности $n$ в пространстве $C^{\infty}(M, E)$. Для отрицательных $\operatorname{ind}\left(P_{1}, 0 \oplus 1_{N}\right)=n<0$ аналогично

$$
P_{1} \sim 0 \oplus\left(1_{N}-(-n)\right)
$$

где $-n$, как и ранее, обозначает конечномерньй проектор размерности $-n>0$. В первом случае мы видим, что в группе Гротендика $K\left(P_{\mathrm{ev}}(M)\right)$ имеет место равенство

$$
\left[P_{1}\right]-\left[0 \oplus 1_{N}\right]=[n]=i(n)
$$

а во втором случае также

$$
\left[P_{1}\right]-\left[0 \oplus 1_{N}\right]=-[-n]=i(n)
$$

Проверим, наконец, точность последовательности (9) в первом члене. Предположим, что для некоторого $n>0$ имеет место равенство

$$
i(n)=0 \text {. }
$$


Тогда из определения групшы Гротендика следует, что для некоторого четного проектора $P \in \operatorname{Even}(M)$ сушествует гомотопия четных проекторов

$$
n \oplus P \sim 0 \oplus P .
$$

Более того, не ограничивая общности, проектор $P$ можно считать единичным: $P=1_{N}$. Обозначим эту непрерывную гомотопию четных проекторов от $n \oplus P$ к $0 \oplus P$ через $P_{t}$. Имеем

$$
\operatorname{ind}\left(P_{0}, P_{1}\right)=n \neq 0 \text {. }
$$

Покажем, что из четности проекторов $P_{t}$ следует, что $\operatorname{ind}\left(P_{0}, P_{1}\right)=0$. Действительно, во-первых, без ограничения общности можно считать, что семейство $\left\{P_{t}\right\}$ состоит из ортогональных проекторов (это следует из того, что пространство всех проекторов стягивается линейно на пространство ортогональных проекторов, ${ }^{3}$ при этом относительный индекс проекторов в формуле (11) не меняется). Рассмотрим далее произвольное периодическое семейство $\left\{A_{t}\right\}_{t=0,1}$ самосопряженных псевдодифференциальных операторов первого порядка с однородными главными символами, равньми на кокасательных сферах $2 \sigma\left(P_{t}\right)-1$ (т.е. положительный спектральный проектор главного символа оператора $A_{t}$ совпадает c $\left.\sigma\left(P_{t}\right)\right)$. Для спектрального потока периодического семейства $\left\{A_{t}\right\}_{t=0,1}$ имеет место когомологическая формула ([4], ср. с [12])

$$
\operatorname{sf}\left\{A_{t}\right\}_{t \in S^{1}}=\left\langle\operatorname{ch}\left(\sigma_{+}\left(A_{t}\right)\right) \pi^{*} \operatorname{Td}\left(T^{*} M \otimes \mathbb{C}\right),\left[S^{1} \times S^{*} M\right]\right\rangle, \quad \pi: S^{1} \times S^{*} M \rightarrow M .
$$

Здесь $\sigma_{+}\left(A_{t}\right) \in \operatorname{Vect}\left(S^{1} \times S^{*} M\right)$ - векторное расслоение, порожденное неотрицательными собственными подпространствами главных символов $\sigma\left(A_{t}\right)$ (в нашем случае $\left.\sigma_{+}\left(A_{t}\right)=\operatorname{Im} \sigma\left(P_{t}\right)\right), \mathrm{Td}-$ класс Тодда расслоения.

На ориентированном многообразии $S^{*} M$ инволюция $(x, \xi) \rightarrow(x,-\xi)$ обращает ориентацию, в то время как класс когомологий

$$
\operatorname{ch}\left(\sigma_{+}\left(A_{t}\right)\right) \pi^{*} \operatorname{Td}\left(T^{*} M \otimes \mathbb{C}\right)
$$

инвариантен относительно этой инволюции. Поэтому спектральњый поток семейства $\left\{A_{t}\right\}_{t=0,1}$ равен нулю:

$$
\operatorname{sf}\left\{A_{t}\right\}_{t=0,1}=0 .
$$

Теперь воспользуемся тем, что семейство проекторов $P_{t}$ является обобщеннылм спектральным сечением [13] для семейства $A_{t}$. Тогда в силу одного из определений спектрального потока (см. [10] или [13]) получаем

$$
\operatorname{sf}\left\{A_{t}\right\}_{t=0,1}=\operatorname{ind}\left(P_{0}, P_{1}\right) \text {. }
$$

Итак,

$$
\operatorname{ind}\left(P_{0}, P_{1}\right)=0
$$

\footnotetext{
${ }^{3}$ Псевдодифференциальнсть ортогонального проекторана подпространство $\operatorname{Im} P$ для произвольного псевдодифференциального проектора $P$ следует из равенства $\operatorname{Im} P=\operatorname{Im} P P^{*}$ и самосопряженности оператора $P P^{*}$.
} 
что находится в противоречии с формулой (11). Точность последовательности (9) установлена.

Заметим теперь, что в терминах последовательности (9) задача о нахождении гомоморфизмов размерности $d$ сводится к задаче о замыкании до коммутативной следуюшей диаграммы

$$
\underset{\substack{\downarrow \\ \mathbb{R}}}{\mathbb{Z}} \stackrel{i}{\longrightarrow} K\left(P_{\mathrm{ev}}(M)\right)
$$

(здесь $\mathbb{Z} \subset \mathbb{R}$ - естественное вложение, а неизвестным является отображение $d$ ). Заметим сначала, что в группах $K\left(P_{\mathrm{ev}}(M)\right)$ и $K\left(P^{*} M\right)$ имеются подгрупшы, порожденные расслоениями на базе $M$ :

$$
\begin{aligned}
& K(M) \longrightarrow K\left(P^{*} M\right), \quad K(M) \stackrel{\alpha}{\longrightarrow} K\left(P_{\mathrm{ev}}(M)\right) \text {, } \\
& {[E] \longrightarrow\left[\pi_{P}^{*} E\right], \quad[E] \longrightarrow\left[1_{C^{\infty}(M, E)}\right] \text {. }}
\end{aligned}
$$

Оба отображения являются мономорфизмами (это следует из существования на нечетномерном многообразии $M$ ненулевого векторного поля), и, кроме того, указанные вложения коммутируют с $q$. Поэтому имеет место частичное расшепление последовательности (9):

$$
0 \longrightarrow \mathbb{Z} \oplus K(M) \stackrel{i \oplus \alpha}{\longrightarrow} K\left(P_{\mathrm{ev}}(M)\right) \stackrel{q}{\longrightarrow} K\left(P^{*} M\right) / K(M) \longrightarrow 0
$$

Факторгруппа $K\left(P^{*} M\right) / K(M)$ в силу [8] целиком состоит из кручения (и кручение есть только в степенях двойки). Поэтому из последовательности (12) вытекает, что произвольная размерность

$$
d: K\left(P_{\mathrm{ev}}(M)\right) \rightarrow \mathbb{R}
$$

однозначно определяется ее заданием на подгруппе $K(M)$ (напомним, что на первом слагаемом в сумме $\mathbb{Z} \oplus K(M)$ размерность $d$ уже задана, см. определение 3$)$ :

$$
\chi: K(M) \rightarrow \mathbb{R}
$$

На этом доказательство теоремы закончено.

ЗАмечАниЕ 1 . Размерность $d$ можно рассматривать как обобщение относительного индекса проекторов, так как для двух четных проекторов $P_{1,2}$ с одинаковыми главњыми символами имеет место формула

$$
\operatorname{ind}\left(P_{1}, P_{2}\right)=d\left(\left[P_{1}\right]-\left[P_{2}\right]\right)
$$

В отношении более явного выражения для размерности $d([P])$ при нулевой нормировке $\chi \equiv 0$ см. $\S 6$, где исследуется связь с $\eta$-инвариантами П. Гилки. 


\section{§ 2. Операторы в подпространствах. Формула индекса для четных подпространств}

Рассмотрим два псевдодифференциальных проектора $P_{1,2}$ нулевого порядка на многообразии $M$

$$
P_{1,2}: C^{\infty}\left(M, E_{1,2}\right) \rightarrow C^{\infty}\left(M, E_{1,2}\right)
$$

и псевдодифференциальный оператор $D$ порядка $m$

$$
D: C^{\infty}\left(M, E_{1}\right) \rightarrow C^{\infty}\left(M, E_{2}\right)
$$

Предположим, что оператор действует в подпространствах, определяемых проекторами, т.е.

$$
D\left(\operatorname{Im} P_{1}\right) \subset \operatorname{Im} P_{2}, \quad P_{2} D P_{1}=D P_{1}
$$

Тогда его сужение

$$
D: \operatorname{Im} P_{1} \rightarrow \operatorname{Im} P_{2}
$$

будем называть оператором, действующим в подпространствах. Известен критерий фредгольмовости операторов такого типа (см. [9] и [2]). Для его формулировки введем понятие главного символа оператора в подпространствах.

ОПРЕДЕЛЕНИЕ 4. Главным символом оператора

$$
D: \operatorname{Im} P_{1} \rightarrow \operatorname{Im} P_{2}
$$

действуюшего в подпространствах, назьвается гомоморфизм расслоений над многообразием $S^{*} M$, задаваемый сужением главного символа оператора $D$

$$
\sigma(D): \operatorname{Im} \sigma\left(P_{1}\right) \rightarrow \operatorname{Im} \sigma\left(P_{2}\right)
$$

Будем называть символ әллиптическим, если он является изоморфизмом, в этом случае оператор $D$ также называется әллиптическим. В этих терминах справедливо следующее предложение, доказательство которого по существу содержится в [2].

ПРЕДЛОЖЕНИЕ 1. Onepamop

$$
D: H^{s}\left(E_{1}\right) \supset \operatorname{Im} P_{1} \rightarrow \operatorname{Im} P_{2} \subset H^{s-m}\left(E_{2}\right)
$$

фредгольмов тогда и только тогда, когда он является әллиптическим.

Порядки соболевских пространств для краткости ниже будут опущены.

Будем рассматривать тройки $\left(D, P_{1}, P_{2}\right)$, задающие эллиптические операторы

$$
D: \operatorname{Im} P_{1} \rightarrow \operatorname{Im} P_{2}
$$

такие, что $P_{1,2}-$ четные проекторы в смысле определения 1. Эти наборы образуют полугруппу $\widetilde{L}_{\mathrm{ev}}(M)$. Тройки вида $\left(1_{N}, 1_{N}, 1_{N}\right)$ будем называть тривиальнымми. 
Две тройки $u_{1}$ и $u_{2}$ будем называть әквивалентными, если для некоторой тривиальной тройки $u_{0}$ сушествует гомотопия

$$
u_{1} \oplus u_{0} \sim u_{2} \oplus u_{0}
$$

Построим группу Гротендика, порожденную полугруппой классов эквивалентных троек

$$
L_{\mathrm{ev}}(M) \stackrel{\text { def }}{=} K\left(\widetilde{L}_{\mathrm{ev}}(M) / \approx\right)
$$

Тогда индекс тройки продолжается до гомоморфизма абелевых групп

$$
\operatorname{ind}_{a}: L_{\mathrm{ev}}(M) \rightarrow \mathbb{Z}
$$

На группе $L_{\mathrm{ev}}(M)$ определим два функционала

$$
\begin{gathered}
\operatorname{ind}_{t}\left(D, P_{1}, P_{2}\right)=\operatorname{ind}_{t}\left(\sigma(D): \operatorname{Im} \sigma\left(P_{1}\right) \rightarrow \operatorname{Im} \sigma\left(P_{2}\right)\right) \\
d\left(D, P_{1}, P_{2}\right)=d\left(P_{1}, P_{2}\right)
\end{gathered}
$$

Прежде всего, рассмотрим отображение

$$
d: L_{\mathrm{ev}}(M) \rightarrow \mathbb{Z}\left[\frac{1}{2}\right], \quad d\left(D, P_{1}, P_{2}\right) \stackrel{\text { def }}{=} d\left(\left[P_{1}\right]-\left[P_{2}\right]\right)
$$

Оно определяется только подпространствами $P_{1}, P_{2}$ и не зависит от оператора, действующего в них. Более того, гомоморфизм (13) не зависит от нормировки $\chi: K(M) \rightarrow \mathbb{R}$. Проверим это утверждение. Во-первых, не ограничивая общности, можно считать, что векторные расслоения $\operatorname{Im} \sigma^{\prime}\left(P_{1,2}\right)$ поднимаются с многообразия $M$ :

$$
\operatorname{Im} \sigma^{\prime}\left(P_{1,2}\right)=\pi_{P}^{*} E_{1,2}
$$

При этом расслоения $E_{1,2}$ будут изоморфными (изоморфизм доставляется эллиптическим символом $\sigma(D)$, опушенным на $M$ при помощи ненулевого векторного поля на многообразии). Таким образом, $K(M)$-составляющие (в смысле последовательности (12)) для проекторов $P_{1,2}$ равны.

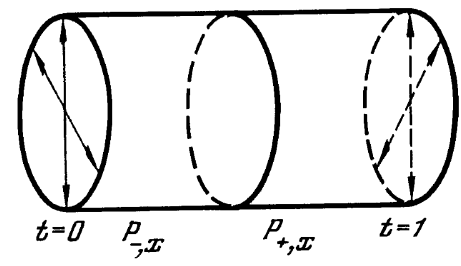

Рис. 1. Слой $\widetilde{P}_{x} M$ 
Построим теперь топологический инвариант троек, продолжающийся до гомоморфизма групп

$$
\operatorname{ind}_{t}: L_{\mathrm{ev}}(M) \rightarrow \mathbb{Q},
$$

которьй определяется только главньм символом оператора. Чтобы это сделать, рассмотрим следуюшее факторпространство $\widetilde{P} M$ (см. рис. 1):

$$
\widetilde{P} M=\left\{S^{*} M \times[0,1] /(x, \pm \xi, 0)\right\} /(x, \pm \xi, 1) .
$$

Это пространство является ориентированньг многообразием, имеющим структуру расслоения над многообразием $M$ со слоем - двумя нечетномерньми проективньми пространствами без маленьких дисков ${ }^{4}$

$$
P_{-, x}=S_{x}^{*} M \times\left[0, \frac{1}{2}\right] /(x, \pm \xi, 0), P_{+, x}=S_{x}^{*} M \times\left[\frac{1}{2}, 1\right] /(x, \pm \xi, 1)
$$

склеенными вдоль их общей гранищы $S_{x}^{*} M \times\{1 / 2\}$ (см. рис. 2$)$

$$
\widetilde{P} M=P_{-} M \bigcup_{S^{*} M} P_{+} M
$$

(получаюшийся слой $\widetilde{P}_{x} M$ является аналогом бутылки Клейна). С помощью естественных проекций в расслоениях со слоем отрезок (см. рис. 2)

$$
P_{\mp} M \rightarrow P^{*} M
$$

продолжим расслоения $\operatorname{Im} \sigma^{\prime}\left(P_{1,2}\right)$ с подпространств $P^{*} M \subset P_{\mp} M$ на все $P_{\mp} M$ соответственно. Тогда изоморфизм $\sigma(D)$ этих расслоений над кокасательными сферами $S^{*} M$ позволяет их склеить, т.е. приводит к векторному расслоению над $\widetilde{P} M$

$$
\sigma\left(D, P_{1}, P_{2}\right) \in \operatorname{Vect}(\widetilde{P} M), \quad\left[\sigma\left(D, P_{1}, P_{2}\right)\right] \in K(\widetilde{P} M) .
$$

На группе $K(\widetilde{P} M)$ можно определить аналог обычного “топологического" индекса (cp. [14])

$$
\operatorname{ind}_{t}\left(D, P_{1}, P_{2}\right)=\left\langle\operatorname{ch}\left[\sigma\left(D, P_{1}, P_{2}\right)\right] \operatorname{Td}\left(T^{*} M \otimes \mathbb{C}\right),[\widetilde{P} M]\right\rangle
$$

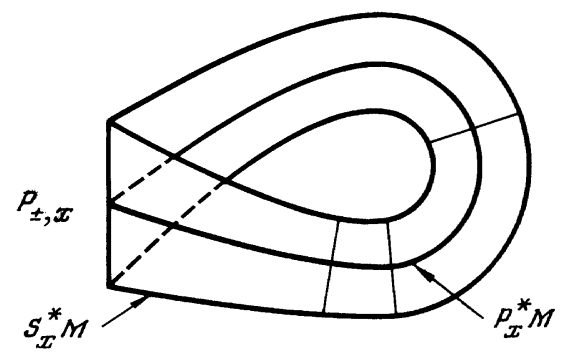

Рис. 2. Сфера как граница проективного пространства без диска

\footnotetext{
${ }^{4}$ Проективное пространство с дыркой является многомерным аналогом листа Мёбиуса, но в нашем случае оно будет ориентируемым.
} 
В этих обозначениях имеет место теорема, в которой устанавливается формула индекса и которая является основным результатом работы.

ТЕОрема 2. Пусть $u=\left(D, P_{1}, P_{2}\right)$ - эллиптический оператор, действующий в подпространствах, определяемых четными псевдодифференциальными проекторами на нечетномерном многообразии М. Тогда справедлива формула индекса

$$
\operatorname{ind}_{a} u=\operatorname{ind}_{t} u+d(u) \text {. }
$$

ДокАЗАТЕЛьство. Левая и правая части формулы (15) являются гомоморфизмами групा

$$
L_{\mathrm{ev}}(M) \rightarrow \mathbb{Q}
$$

Для доказательства равенства этих гомоморфизмов в силу того обстоятельства, что группа рациональных чисел не имеет кручения, достаточно проверить равенство только на элементах групшы $L_{\mathrm{ev}}(M)$, которые ее рационально порождают. Из точности последовательности (12) и того, что группа $K\left(P^{*} M\right) / K(M)$ целиком состоит из кручения, следует, что для произвольной тройки $\left(D, P_{1}, P_{2}\right)$ проекторы $P_{1,2}$ могут быть рационально прогомотопированы (т.е. гомотопна прямая сумма вида $\left.P_{1,2} \oplus \cdots \oplus P_{1,2}\right)$ к проекторам, которые отличаются от проекторов на пространства сечений некоторых расслоений на конечномерные проекторы. Поэтому в качестве элементов, рационально порождающих группу $L_{\mathrm{ev}}(M)$, можно взять тройки вида

$$
u=\left(\left(\begin{array}{cc}
0 & 0 \\
D & 0
\end{array}\right), 1_{E} \oplus n, m \oplus 1_{F}\right),
$$

где $E, F \in \operatorname{Vect}(M)$, через $n, m$ обозначены конечномерные проекторы, а оператор

$$
D: C^{\infty}(M, E) \rightarrow C^{\infty}(M, F)
$$

- обычный псевдодифференциальный оператор в пространствах. Проверим, что для таких операторов выполнено (15). Рассмотрим соотношения

$$
\begin{gathered}
\operatorname{ind}_{a} u=\operatorname{ind} D+n-m, \\
d(u)=n-m+d\left(\left[1_{C}^{\infty}(M, E)\right]-\left[1_{C^{\infty}(M, F)}\right]\right)=n-m
\end{gathered}
$$

(во втором соотношении пользуемся ненулевым векторньм полем для установления изоморфности расслоений $E$ и $F$ ). Поэтому при проверке соотношения (15) для тройки $u$ достаточно проверить равенство

$$
\text { ind } D=\operatorname{ind}_{t}\left(D, 1_{C^{\infty}(M, E)}, 1_{C^{\infty}(M, F)}\right) \text {. }
$$

Покажем, что правая часть этой формулы является незначительной модификацией обычной формулы Атьи-Зингера для индекса эллиптического оператора $D$. Для этого перепишем формулу Атьи-Зингера в виде (см. [14])

$$
\text { ind } D=\left\langle\operatorname{ch}[\sigma(D)] \pi^{*} \operatorname{Td}\left(T^{*} M \otimes \mathbb{C}\right),[\widetilde{B} M]\right\rangle, \quad \widetilde{B} M=B_{-} M \bigcup_{S^{*} M} B_{+} M
$$


где $B_{ \pm} M$ - расслоения единичных кокасательных дисков на многообразии $M$. Рассмотрим далее оба выражения в формулах (17) и (14) как интегралы по многообразиям от характеристических классов, представленных в виде дифференциальных форм с помощью связностей в соответствуюших векторных расслоениях. Многообразия $\widetilde{B} M$ и $\widetilde{P} M$ диффеоморфны в окрестности $S^{*} M \times\{1 / 2\}$. Заметим также, что на этих многообразиях действует обращающая ориентацию инволюция $(x, \xi, t) \rightarrow(x,-\xi, t)$, сохраняюшая половинки $P_{ \pm}$и $B_{ \pm}$. Следовательно, для связностей в векторном расслоении $\sigma\left(D, P_{1}, P_{2}\right)$ над $\widetilde{P} M$, которые являются инвариантными относительно инволюции вне некоторой окрестности кокасательных сфер $S^{*} M \times\{1 / 2\}$, вклады в формулу (16) от соответствующих областей равны нулю (так как подынтегральные выражения инвариантны относительно инволюции, которая обрашает ориентацию), аналогичное сокрашение происходит и с $\widetilde{P} M$. Остаюшийся вклад, возникающий при интегрировании по окрестности $S^{*} M \times\{1 / 2\}$, является одинаковым для обеих формул, так как подынтегральные выражения поточечно совпадают.

Таким образом, для случая проекторов с единичными главными символами формула индекса доказана. Как было отмечено выше, такие операторы в подпространствах рационально порождают всю группу $L_{\mathrm{ev}}(M)$. Поэтому формула индекса, а вместе с ней и теорема, доказана в общем случае.

Приведем два важных замечания, касающихся топологического слагаемого ind $t$ в формуле индекса.

ЗАМЕчАНИЕ 2. При определении группы стабильно гомотопически эквивалентных троек $L_{\mathrm{ev}}(M)$ можно было бы пойти дальше и профакторизовать эту группу по тройкам $(P, P, P)$ с произвольным четным проектором $P$, которые, очевидно, не дают вклада в индекс. Получаюшуюся группу обозначим тем же символом $L_{\mathrm{ev}}$. В этом случае вместо группы $K(\widetilde{P} M)$, принимающей главный символ задачи, надо брать ее факторгруппу $K(\widetilde{P} M) / K\left(P^{*} M\right)$ и соответствующее отображение, задаваемое главным символом

$$
L_{\mathrm{ev}}(M) \rightarrow K(\widetilde{P} M) / K\left(P^{*} M\right) .
$$

Последняя группа является аналогом $K$-функтора, отвечающего разностной конструкции обычной эллиптической теории $K_{c}\left(T^{*} M\right)$ в силу естественного изоморфизма

$$
K(\widetilde{P} M) / K\left(P^{*} M\right) \simeq K_{c}\left({ }^{p} T^{*} M\right),
$$

где ${ }^{p} T^{*} M=P_{-} M \bigcup_{S^{*} M}\left\{T^{*} M \cap\{|\xi| \geqslant 1\}\right\}$. В этом случае топологический индекс

$$
K_{c}\left({ }^{p} T^{*} M\right) \rightarrow \mathbb{Q}
$$

задается той же формулой (14).

В самом деле, это утверждение является следствием изоморфизма (18), который в свою очередь геометрически очевиден: факторгруппа в левой части изоморфна относительной групше

$$
K(\widetilde{P} M) / K\left(P^{*} M\right) \simeq K\left(\widetilde{P} M, P_{+} M\right),
$$

а некомпактные пространства $\widetilde{P} M \backslash P_{+} M{ }^{p} T^{*} M$ собственно гомеоморфны (т.е. гомеоморфизм задается собственным отображением). 
ЗАмЕчАнИЕ 3 . Топологический индекс троек ind $t$ редуцируется к топологическому индексу обычного эллиптического оператора. Для тройки $\left(D, P_{1}, P_{2}\right)$ рассмотрим символ

$$
\sigma(D)^{-1}(x,-\xi) \sigma(D)(x, \xi) \oplus 1: \pi_{S}^{*} E_{1} \rightarrow \pi_{S}^{*} E_{1}, \quad \pi_{S}: S^{*} M \rightarrow M
$$

где прямая сумма символов рассматривается по отношению к разложению расслоения

$$
\pi_{S}^{*} E=\operatorname{Im} \sigma\left(P_{1}\right)(x, \xi) \oplus \operatorname{Im}\left(1-\sigma\left(P_{1}\right)(x, \xi)\right) .
$$

При этом имеет место равенство

$$
\operatorname{ind}_{t}\left(D, P_{1}, P_{2}\right)=\frac{1}{2} \operatorname{ind}_{t}\left(\sigma(D)^{-1}(x,-\xi) \sigma(D)(x, \xi) \oplus 1\right)
$$

где в правой части равенства стоит топологический индекс обычного символа в пространствах.

Для доказательства формулы (19) заметим, что в начале этого параграфа мы могли взять эту формулу в качестве определения ind $d_{t}\left(D, P_{1}, P_{2}\right)$. После этого в доказательстве теоремы об индексе нужно было бы проверить только, что для “классического" (т.е. действующего в сечениях расслоений на базе) оператора $D$ выражение в правой части формулы (19) равно (топологическому) индексу оператора $D$. Действительно, в этом случае

$$
\begin{aligned}
\operatorname{ind}_{t} & \left(\sigma(D)^{-1}(x,-\xi) \sigma(D)(x, \xi) \oplus 1\right) \\
\quad= & \operatorname{ind}_{t}\left(\sigma(D)^{-1}(x,-\xi)\right)+\operatorname{ind}_{t}(\sigma(D)(x, \xi)) \\
= & -\operatorname{ind}_{t}(\sigma(D)(x,-\xi))+\operatorname{ind}_{t}(\sigma(D)(x, \xi)) \\
\quad= & 2 \operatorname{ind}_{t}(\sigma(D)(x, \xi))=2 \operatorname{ind} D .
\end{aligned}
$$

В этой цепочке равенств участвуют только символы в классическом смысле, $\mathrm{a} \mathrm{ind}_{t}$ - обычный топологический индекс; в предпоследнем равенстве мы воспользовались тем, что инволюция $(x, \xi) \rightarrow(x,-\xi)$ многообразия $S^{*} M$ обрашает ориентацию, а последнее равенство состоит в применении теоремы Атьи-Зингера.

\section{§ 3. Общие краевые задачи}

Напомним кратко определение общих краевых задач [2].

Пусть $M$ - компактное гладкое многообразие с краем. Рассмотрим эллиптический дифференциальньй оператор $D$ порядка $m$, действующий в пространствах сечений векторных расслоений на $M$

$$
D: C^{\infty}(M, E) \rightarrow C^{\infty}(M, F)
$$

Для некоторой воротниковой окрестности края с нормальной координатой $t$ (внутренность многообразия отвечает положительным значениям) будем рассматривать джеты сечений расслоений в нормальном направлении

$$
j_{\partial M}^{m-1} u=\left(u(0),\left.\frac{\partial}{\partial t} u\right|_{t=0}, \ldots,\left.\frac{\partial^{m-1}}{\partial t^{m-1}} u\right|_{t=0}\right): C^{\infty}(M, E) \rightarrow C^{\infty}\left(\partial M, E^{m}\right) .
$$


Здесь

$$
E^{m}=\underbrace{E \oplus E \oplus \cdots \oplus E}_{m \text { раз }} .
$$

Общей краевой задачей назьвается система уравнений вида

$$
\left\{\begin{array}{l}
D u=f, \quad u \in C^{\infty}(M, E), \quad f \in C^{\infty}(M, F) \\
B j_{\partial M}^{m-1} u=g \in \operatorname{Im} P, \quad \operatorname{Im} P \subset C^{\infty}(\partial M, G)
\end{array}\right.
$$

При этом псевдодифференциальньй оператор $P$

$$
P: C^{\infty}(\partial M, G) \rightarrow C^{\infty}(\partial M, G)
$$

имеет нулевой порядок и является проектором $P^{2}=P$, граничный оператор $B$

$$
B: C^{\infty}\left(\partial M, E^{m}\right) \rightarrow C^{\infty}(\partial M, G)
$$

также является псевдодифференциальньм и действует в подпространство $\operatorname{Im} P$ : $P B=B$. Краевую задачу (20) будем обозначать через $(D, B, P)$. Классические краевые задачи получаются при рассмотрении единичных проекторов $P=1$ в формулах (20).

Для формулировки критерия фредгольмовости таких краевых задач рассмотрим для каждой точки $\left(x, \xi^{\prime}\right)$ расслоения кокасательных сфер к краю $S^{*} \partial M$ обыкновенное дифференциальное уравнение с постоянными коэффициентами на прямой $t \in \mathbb{R}$

$$
\sigma(D)\left(x, 0,-i \frac{d}{d t}, \xi^{\prime}\right) u(t)=0
$$

(здесь $\sigma(D)$ - главный символ оператора $D$ ) и обозначим подпространство начальных данных Коши ограниченных решений при $t \rightarrow \pm \infty$ через $L^{\mp}\left(x, \xi^{\prime}\right)$. Тогда для эллиптических операторов $D$ имеет место разложение

$$
E_{x}^{m}=L^{+}\left(x, \xi^{\prime}\right) \oplus L^{-}\left(x, \xi^{\prime}\right),
$$

более того, семейства $L^{ \pm}\left(x, \xi^{\prime}\right)$ в этом случае образуют гладкие векторные подрасслоения

$$
L^{ \pm} \subset \pi^{*} E^{m}, \text { где } \pi: S^{*} \partial M \rightarrow \partial M .
$$

ЗАмечаниЕ 4. Нетрудно проверить, что разложение (21) является необходимым и достаточным условием эллиптичности оператора $D$ на крае.

Имеет место следующий критерий фредгольмовости общих краевых задач [2]. 
Теорема 3. Краевая задача $(D, B, P)$ определяет фредгольмов оператор npu $s>m-\frac{1}{2}$

$$
\left(\begin{array}{c}
D \\
B j_{\partial M}^{m-1}
\end{array}\right): H^{s}(M, E) \rightarrow{ }^{H^{s-m}(M, F)} \quad \bigoplus_{\operatorname{Im} P}^{\oplus}, \quad \operatorname{Im} P \subset H^{\delta}(\partial M, G)
$$

тогда и только тогда, когда оператор $D$ является эллиптическим и ограничение главного символа граничного оператора $B$ на подрасслоение $L^{-}$задает изоморфизм

$$
\sigma(B): L^{-}(D) \rightarrow \operatorname{Im} \sigma(P)
$$

Предполагается, что порядки компонент оператора $B$ по отночению $\kappa$ джету $j_{\partial M}^{m-1}$ согласованы с индексами $s, \delta$ пространств Соболева.

Класс краевых задач для дифференциальных операторов достаточно узок для проведения гомотопий эллиптических символов. Оказывается, что следующее ниже несложное обобшение этого класса позволяет производить гомотопии, т.е. применять топологические методы для решения проблемы индекса краевых задач.

В этой работе мы будем использовать операторы, дифференциальные по нормальной переменной $t$. А именно, введем в рассмотрение операторы $D$ на многообразии $M$, которые, во-первых, в окрестности края имеют вид

$$
D=\sum_{k=0}^{m} A_{k}(t)\left(-i \frac{\partial}{\partial t}\right)^{k}
$$

где $A_{k}(t)$ - гладкие семейства псевдодифференциальных операторов порядка $m-k$, причем оператор $A_{m}(t)$ является гомоморфизмом расслоений. Во-вторых, на всем многообразии оператор $D$ должен быть представлен конструкцией псевдодифференциального оператора с непрерывным символом (см. [15], [16] или [6]). Заметим, что необходимость рассмотрения операторов с непрерывными символами происходит из того, что уже символ оператора (22) не является, вообще говоря, гладким. ${ }^{5}$

Для операторов из только что описанного класса общие краевые задачи ставятся точно так же, как и в обычном случае (20). Сохраняются определение пространств $L^{ \pm}$и критерий фредгольмовости (теорема 3$)$.

Рассмотрим пример краевых задач для операторов вида (22). Именно приводимые ниже задачи и позволяют (в классическом случае) свести проблему индекса к известному случаю замкнутого многообразия.

ПримеР 1. Пусть расслоение $E$ в окрестности края $\partial M$ разложено в сумму двух подрасслоений

$$
\left.E\right|_{U_{\partial M}}=E_{+} \oplus E_{-}
$$

Рассмотрим в расслоениях $\left.E_{ \pm}\right|_{\partial м}$ эллиптические операторы $\Lambda_{ \pm}$первого порядка с главными символами, равными $\left|\xi^{\prime}\right|$. Зафиксируем также оператор первого порядка $\Lambda$ с главньм символом $|\xi|$, действуюший в расслоении $E$ на всем многообразии.

\footnotetext{
5 Это известная проблема непсевдодифференциальности тензорных произведений (см. [15]). Здесь это, например, произведение операторов $\partial / \partial t$ и $A_{1}(t)$.
} 
В соответствии с разложением (23) определим в окрестности края следующий эллиптический оператор первого порядка

$$
D=\left(-i \frac{\partial}{\partial t}+i \Lambda_{+}\right) \oplus\left(+i \frac{\partial}{\partial t}+i \Lambda_{-}\right): C^{\infty}\left(U_{\partial M}, E\right) \rightarrow C^{\infty}\left(U_{\partial M}, E\right) .
$$

Очевидно, что для его главного символа на краю имеет место равенство

$$
L^{-}(D)=0 \oplus \pi^{*} E_{-} .
$$

Таким образом, следуюшее краевое условие для этого оператора является эллиптическим

$$
\left.u_{-}\right|_{\partial M}=g \in C^{\infty}\left(\partial M, E_{-}\right) \text {для } u=\left(u_{+}, u_{-}\right) \in C^{\infty}\left(U_{\partial M}, E_{+} \oplus E_{-}\right) .
$$

Этот оператор нетрудно продолжить на все многообразие. Для этого рассмотрим гладкую срезаюшую функцию на $M: 0 \leqslant \chi(t) \leqslant 1$, которая тождественно равна 1 при $0 \leqslant t \leqslant 1 / 3$ и обрашается в нуль при $t \geqslant 2 / 3$. Тогда требуемое продолжение получается, например, по формуле

$$
D=\chi(t)\left[\left(-i \frac{\partial}{\partial t}+i \Lambda_{+}\right) \oplus\left(+i \frac{\partial}{\partial t}+i \Lambda_{-}\right)\right]+(1-\chi(t)) i \Lambda .
$$

Оператор $D$ с краевым условием (25) задает эллиптическую краевую задачу. Хорошо известно, что эта краевая задача имеет нулевой индекс. Этот факт можно доказать, заметив, что семейство краевых задач

$$
D+i p
$$

является эллиптическим с параметром $p$ по Аграновичу-Вишику [17] в полуплоскости $\operatorname{Re} p>0$ и поэтому обратимо при больших значениях $p$; впрочем, обратимость семейства $D+i p$ может быть доказана и непосредственно (см. [6]). Подправив оператор $D$ на конечномерньй оператор, можно считать, что сам $D$ является обратимым.

В следующем параграфе при проведении редукций краевых задач нам будет удобно гомотопировать не непосредственно оператор $D$ как оператор на многообразии $M$, а только лишш его ограничение на малую окрестность края. Более точно, будут предъявлены гомотопии вида

$$
\sum_{k=0}^{m} A_{k}(t, \tau)\left(-i \frac{\partial}{\partial t}\right)^{k}
$$

определенные при малых значениях $t$, например, при $t<1$. Параметром гомотопии является $\tau \in[0,1]$. Легко заметить, что по такой гомотопии в окрестности края можно построить гомотопию оператора $D$ на всем многообразии. Искомая гомотопия оператора $D$ при $t \geqslant 1$ является постоянной, а в остающейся части воротниковой окрестности края она равна (для срезающей функции $\chi(t)$ из примера, рассмотренного выше)

$$
D_{\tau}=\sum_{k=0}^{m} A_{k}(t, \tau \chi(t))\left(-i \frac{\partial}{\partial t}\right)^{k}
$$




\section{§4. От общих краевых задач к спектральным}

В настоящем параграфе мы, пользуясь техникой теории индекса классических краевых задач [6], покажем, что произвольная общая краевая задача канонически редуцируется к некоторой спектральной задаче. Более того, в процессе редукции пространство граничных данных, определяемое проектором $P$, не меняется. Эту редукцию разобьем на несколько шагов.

Кратко прокомментируем соответствуюшие построения. Шаги 1 и 2 редукции являются вспомогательными в том смысле, что в них не затрагивается оператор краевых условий $B$. На третьем шаге, являющемся основой конструкции, производится гомотопия граничного оператора $B$ к тривиальному.

Шаг 1. Редукция к операторам первого порядка. Проблема индекса краевой задачи $(D, B, P)$ для оператора порядка $m$ редуцируется к аналогичной задаче, но для некоторого оператора первого порядка в силу следуюшей теоремы.

ТЕОРема 4. Эллиптическая краевая задача $(D, B, P), \operatorname{deg} D=m$, стабильно гомотопна композичии краевых задач

$$
\left(D^{\prime}, B^{\prime}, P\right) \circ m D_{+}^{m-1}, \quad m D_{+}^{m-1} \stackrel{\text { def }}{=} \underbrace{D_{+}^{m-1} \oplus \cdots \oplus D_{+}^{m-1}}_{m \text { раз }}
$$

где оператор $D_{+}-$обратимый оператор первого порядка из примера 1 (для расслоений $\left.E_{-}=0, E=E_{+}\right)$, для которого не требуется краевых условий, а $\left(D^{\prime}, B^{\prime}, P\right)$ - краевая задача для оператора первого порядка. При этом в процессе гомотопии проектор $P$ не меняется.

ДокАЗАтЕЛьство. Рассмотрим прямую сумму краевых задач

$$
(D, B, P) \oplus \bigoplus_{1}^{m-1} D_{+}^{m}
$$

Ее индекс совпадает с индексом исходной задачи, так как оператор $D_{+}$обратим. Представим оператор $D$ в виде

$$
D=\sum_{k=0}^{m} D_{k}(t)\left(-i \frac{\partial}{\partial t}-i \Lambda\right)^{k}\left(-i \frac{\partial}{\partial t}+i \Lambda\right)^{m-k} \equiv \sum_{k=0}^{m} D_{k}(t) D_{-}^{k} D_{+}^{m-k}
$$

где оператор $\Lambda$ с главным символом $\left|\xi^{\prime}\right|$ действует в расслоении $E$ в окрестности края. Рассмотрим следующую гомотопию операторов (в этих матрицах для краткости опускаем аргумент $t$ у коэффициентов $\left.D_{k}(t)\right)$

$$
\begin{aligned}
D_{\tau}= & \left(\begin{array}{cccc}
D-\tau^{m} D & 0 & \ldots & 0 \\
0 & D_{+}^{m} & \ldots & 0 \\
0 & 0 & D_{+}^{m} & \ldots \\
0 & 0 & \ldots & D_{+}^{m}
\end{array}\right) \\
& +\left(\begin{array}{cccc}
\tau^{m} D_{0} D_{+}^{m} & \tau^{m-1} D_{1} D_{+}^{m} & \ldots & \tau D_{m-1} D_{+}^{m}+\tau D_{m} D_{-} D_{+}^{m-1} \\
-\tau D_{-} D_{+}^{m-1} & 0 & \ldots & 0 \\
0 & -\tau D_{-} D_{+}^{m-1} & \ldots & \ldots \\
0 & 0 & \ldots & 0
\end{array}\right)
\end{aligned}
$$


В начальньй момент $\tau=0$ имеем $D_{\tau=0}=D \oplus \bigoplus_{1}^{m-1} D_{+}^{m}$. С другой стороны, при $\tau=1$ получаем требуемую в теореме факторизацию

$$
D_{\tau=1}=\left(\begin{array}{cccc}
D_{0}(t) D_{+} & D_{1}(t) D_{+} & \ldots & D_{m-1}(t) D_{+}+D_{m}(t) D_{-} \\
-D_{-} & D_{+} & 0 & 0 \\
0 & -D_{-} & D_{+} & \ldots \\
0 & 0 & \ldots & D_{+}
\end{array}\right) \circ m D_{+}^{m-1}
$$

Проверим, что для $\tau \in[0,1]$ оператор $D_{\tau}$ эллиптичен. Найдем сначала подпространства $L^{-}\left(D_{\tau}\right)$. Пусть $U=\left(U_{0}(t), U_{1}(t), \ldots, U_{m-1}(t)\right)$ - ограниченное при $t \rightarrow+\infty$ решение уравнения

$$
\sigma\left(D_{\tau}\right)\left(x, 0,-i \frac{d}{d t}, \xi^{\prime}\right) U=0
$$

Надо отметить, что производные решения $U$ также ограничены, так как эта функция является решением дифференциального уравнения с постоянными коэффициентами.

Записьвая приведенное выше уравнение покомпонентно, получаем систему

$$
\left\{\begin{array}{l}
\left(1-\tau^{m}\right) \sigma(D)\left(-i \frac{d}{d t}\right) U_{0}+\left(-i \frac{d}{d t}+i\right)^{m}\left(d_{0} \tau^{m} U_{0}+\cdots+d_{m-1} \tau U_{m-1}\right) \\
\quad+\tau\left(-i \frac{d}{d t}+i\right)^{m-1}\left(-i \frac{d}{d t}-i\right) d_{m} U_{m-1}=0 \\
\left(-i \frac{d}{d t}+i\right)^{m} U_{j}=\tau\left(-i \frac{d}{d t}+i\right)^{m-1}\left(-i \frac{d}{d t}-i\right) U_{j-1}, \quad 0<j<m,
\end{array}\right.
$$

где через $d_{j}$ обозначены главные символы операторов $D_{j}(0)$. В силу того, что уравнение на полупрямой

$$
\left(-i \frac{d}{d t}+i\right) u=0
$$

не имеет ограниченных решений, сокрашаем на этот оператор в системе (28) все уравнения, кроме первого. Таким образом, получаем

$$
\left(-i \frac{d}{d t}+i\right) U_{j}=\tau\left(-i \frac{d}{d t}-i\right) U_{j-1}
$$

Последовательно подставляя эти соотношения одно в другое, имеем

$$
\left(-i \frac{d}{d t}+i\right)^{j} U_{j}=\tau^{j}\left(-i \frac{d}{d t}-i\right)^{j} U_{0}
$$

Учитывая эти выражения в первом уравнении системы (28), обнаруживаем, что оно сводится к требованию

$$
\sigma(D)\left(-i \frac{d}{d t}\right) U_{0}=0
$$


Отсюда следует эллиптичность оператора $D_{\tau}$ : уравнение $(29)$ ограниченных на всей прямой решений не имеет, т.е. разложение (21) действительно имеет место.

Таким образом, получено следуюшее описание подрасслоения $L^{-}\left(D_{\tau}\right)$. Проекция на первое слагаемое в сумме

$$
E \oplus \bigoplus_{1}^{m-1} E \stackrel{\mathrm{pr}}{\longrightarrow} E
$$

индуцирует изоморфизм расслоений

$$
L^{-}\left(D_{\tau}\right) \stackrel{\mathrm{pr}}{\longrightarrow} L^{-}(D)
$$

прообраз элемента $u \in L^{-}(D)$ при этом отображении задается формулой

$$
\begin{gathered}
U=\left(U_{0}, \ldots, U_{m-1}\right) \\
U_{0}=u, \quad\left(-i \frac{d}{d t}+i\right)^{j} U_{j}=\tau^{j}\left(-i \frac{d}{d t}-i\right)^{j} U_{0}
\end{gathered}
$$

Так же, как выше был переписан оператор $D$, разложим оператор граничных условий

$$
B j_{\partial M}^{m-1}=\left.\sum_{k=0}^{m-1} B_{k}\left(-i \frac{\partial}{\partial t}-i \Lambda\right)^{k}\left(-i \frac{\partial}{\partial t}+i \Lambda\right)^{m-1-k}\right|_{t=0}
$$

Тогда главный символ граничного условия

$$
B j_{\partial M}^{m-1} \circ \mathrm{pr}: C^{\infty}\left(M, E \oplus \bigoplus_{1}^{m-1} E\right) \rightarrow C^{\infty}(\partial M, G)
$$

на подпространстве $L^{-}\left(D_{\tau=1}\right)$ в силу равенств $(30)$ имеет факторизацию

$$
\sigma\left(B^{\prime}\right) j_{\partial м} \circ\left(-i \frac{d}{d t}+i\right)^{m-1}, \quad \text { где }\left(\sigma\left(B^{\prime}\right) j_{\partial M}\right) U=\sum_{k=0}^{m-1} b_{k} U_{k}(0)
$$

Таким образом, гомотопия эллиптических краевых задач

$$
\left(D_{\tau}, B \circ \operatorname{pr}, P\right), \quad \tau \in[0,1]
$$

соединяет исходную задачу (27) с краевой задачей, являющейся требуемой в теореме композицией

$$
\left(D_{\tau=1}, B \circ \operatorname{pr}, P\right)=\left(D^{\prime}, B^{\prime}, P\right) \circ m D_{+}^{m-1} .
$$

На этом доказательство завершено. 
Шаг 2. Выражение оператора на границе через проектор Кальдерона. На этом шаге мы покажем, что оператор первого порядка в окрестности края редуцируется к оператору, однозначно определяемому подрасслоением $L^{-}(D)$.

Рассмотрим краевую задачу для эллиптического оператора первого порядка

$$
D=-i A_{1} \frac{\partial}{\partial t}+A_{0}: C^{\infty}(M, E) \rightarrow C^{\infty}(M, F)
$$

Коэффициент $A_{1}$ является по предположению изоморфизмом расслоений в окрестности края. Отождествим с его помощью расслоения $E$ и $F$. Тогда оператор $D$ примет вид

$$
D=-i \frac{\partial}{\partial t}+A
$$

Из эллиптичности $D$ следует, что собственные значения главного символа оператора $A$ при ненулевых значениях кокасательной к краю переменной $\xi^{\prime}$ имеют ненулевые мнимые части. Простое вычисление показывает, что подрасслоение $L^{-}(D)$ порождено спектральными подпространствами символа $\sigma(A)$ с отрицательными мнимыми частями собственных значений. Обозначим соответствующий спектральньй проектор через $q$

$$
q: \pi^{*} E \rightarrow \pi^{*} E, \quad \operatorname{Im} q=L^{-}(D)
$$

Рассмотрим также произвольный псевдодифференциальный оператор $Q$ (не обязательно проектор) с главным символом, равным $q$. Такие операторы называют проекторами Кальдерона оператора $D$ (см. [6]).

Рассмотрим линейную гомотопию операторов

$$
D_{\tau}=-i \frac{\partial}{\partial t}+(1-\tau) A-i \tau(2 Q-1) \Lambda
$$

здесь $\Lambda$ - оператор с главным символом, равным $\left|\xi^{\prime}\right|$. В силу того, что проектор $q\left(\xi^{\prime}\right)$ является спектральным проектором символа $\sigma(A)\left(\xi^{\prime}\right)$, получаем, что собственные значения символа

$$
(1-\tau) \sigma(A)\left(\xi^{\prime}\right)-i \tau(2 q-1)\left|\xi^{\prime}\right|
$$

равны соответственно (здесь $\lambda$ - произвольное собственное значение символа $\sigma(A)$ )

$$
(1-\tau) \lambda+i \tau \operatorname{sign}(\operatorname{Im} \lambda)
$$

Отсюда следует эллиптичность оператора $D_{\tau}$ и независимость подрасслоения $L^{-}\left(D_{\tau}\right)$ от параметра $\tau$.

Таким образом, мы проводим гомотопию краевых задач

$$
\left(D_{\tau}, B, P\right), \quad \tau \in[0,1]
$$


в результате которой главньй символ получающегося оператора $D$ на крае определяется символом проектора Кальдерона $Q$

$$
D_{\tau=1}=-i \frac{\partial}{\partial t}-i(2 Q-1) \Lambda
$$

В завершение этого шага рассмотрим ортогональный проектор $q^{\prime}$ на подрасслоение $L^{-}(D)$ и псевдодифференциальный проектор $Q^{\prime}$ с главным символом $q^{\prime}$. Тогда линейная гомотопия

$$
-i \frac{\partial}{\partial t}-i\left(2\left(\tau Q^{\prime}+(1-\tau) Q\right)-1\right) \Lambda
$$

приводит при $\tau=1$ к оператору

$$
-i \frac{\partial}{\partial t}-i\left(2 Q^{\prime}-1\right) \Lambda
$$

главный символ которого однозначно определяется подрасслоением $L^{-}(D)$. Поэтому можно считать, что символ $q$ проектора Кальдерона является ортогональным проектором на подрасслоение $L^{-}(D)$.

Шаг 3. Редукция проектора Кальдерона к проектору граничных данных. В этом пункте мы произведем гомотопию операторов $D$ и $B$ краевой задачи $(D, B, P)$, в результате которой граничный оператор $B$ преобразуется из оператора, символ которого был изоморфизмом подрасслоений

$$
L^{-}(D) \stackrel{\sigma(B)}{\longrightarrow} \operatorname{Im} \sigma(P)
$$

в тождественньй оператор. В частности, подрасслоение $L^{-}(D)$ будет продеформировано в $\operatorname{Im} \sigma(P)$. Формула (31) показывает, что для построения такой гомотопии краевых задач достаточно гомотопировать только символ проектора Кальдерона $q$, а также символ оператора граничных условий $\sigma(B)$. Из теории векторных расслоений хорошо известно, что изоморфиые подрасслоения (в нашем случае это $L^{-}(D)$ и $\left.\operatorname{Im} \sigma(P)\right)$ могут быть продеформированы одно в другое как подрасслоения в некотором объемлющем расслоении (при условии, что его размерность достаточно велика). Выпишем для нашей ситуации явную формулу такой гомотопии.

Реализуем расслоение $G \in \operatorname{Vect}(\partial M)$ как подрасслоение в некотором тривиальном расслоении $\mathbb{C}^{N}$

$$
G \stackrel{\alpha}{\subset} \mathbb{C}^{N}
$$

Напомним, что граничные данные краевой задачи принадлежат пространству сечений этого расслоения: $\operatorname{Im} P \subset C^{\infty}(\partial M, G)$.

Рассмотрим прямую сумму краевых задач

$$
(D, B, P) \oplus D_{+},
$$

где обратимый оператор $D_{+}-$оператор из примера 1 , отвечающий расслоениям $E_{+}=E=\mathbb{C}^{N}$. В этих обозначениях искомая гомотопия подрасслоений

$$
L^{-}(D) \oplus 0, \quad 0 \oplus \operatorname{Im} \sigma(P) \subset \pi^{*}\left(E \oplus \mathbb{C}^{N}\right)
$$


является гомотопией поворота на $90^{\circ}$ при помощи символа $\sigma(B)$. Более точно, для угла $\tau \in[0, \pi / 2]$ определим подрасслоение

$$
L^{-}(\tau) \subset \pi^{*}\left(E \oplus \mathbb{C}^{N}\right),
$$

слой которого в каждой точке $\left(x, \xi^{\prime}\right) \in S^{*} \partial M$ порожден векторами вида

$$
v_{\tau}=(v \cos \tau, \sigma(B) v \sin \tau) \text { для всех } v \in L^{-}(D) .
$$

Главный символ граничного оператора $B$ будем гомотопировать по формуле

$$
\sigma\left(B_{\tau}\right) v_{\tau} \stackrel{\text { def }}{=} \sigma(B) v
$$

Из формулы (32), очевидно, получаем, что в конце гомотопии при $\tau=\pi / 2$

$$
L^{-}(\pi / 2)=0 \oplus \operatorname{Im} \sigma(P), \quad \sigma\left(B_{\pi / 2}\right)=\operatorname{Id} .
$$

Таким образом, оператор $\left(D_{\pi / 2}, B_{\pi / 2}, P\right)$ является требуемой спектральной краевой задачей.

На этом редукция общей краевой задачи к спектральной задаче вида (2) полностью завершена.

\section{§5. Индекс четных задач}

В этом параграфе будет получена формула индекса для обших краевых задач (2) при дополнительном условии четности проектора $P .6$ Эту формулу мы докажем сведением к некоторой классической краевой задаче. Для этого изучим связь между четными и классическими задачами.

Рассмотрим группу Гротендика $K\left(P_{\mathrm{ev}}(X)\right)$, порожденную абелевой полугруппой классов эквивалентности четных проекторов (см. формулу (8)). В ней имеется подгруппа, порожденная единичными проекторами. Соответствующая факторгруппа есть $K\left(P_{\mathrm{ev}}(X)\right) / K(X)$.

Спектральную краевую задачу вида (2) на многообразии $M$ будем обозначать через $\mathscr{D}=(D, P)$. Такую задачу будем называть четной, если проектор $P$ является четным проектором на крае $\partial M$. Введем отношение эквивалентности четных задач. Именно, будем считать задачи $\mathscr{D}_{1}$ и $\mathscr{D}_{2}$ эквивалентными, если существует четная гомотопия

$$
\mathscr{D}_{1} \oplus D_{+} \sim D_{+} \oplus \mathscr{D}_{2}
$$

где $D_{+}$, как и в предыдушем параграфе, обратимый оператор из примера 1 при выборе расслоений $E=E_{+}$. Группу Гротендика четных задач обозначим через $K\left(\mathscr{D}_{\text {ev }}(M)\right)$. В ней имеется подгруппа, порожденная классическими краевыми задачами из примера 1 для произвольных векторных расслоений $E$ на $M$ и $E_{+}$на $\partial M$ соответственно. Факторгруппу обозначим через

$$
K\left(\mathscr{D}_{\mathrm{ev}}(M)\right) /(K(\partial M) \oplus K(M))
$$

\footnotetext{
${ }^{6} \mathrm{~B}$ сочетании с редукциями предыдущего параграффа из этого следует формула индекса краевых задач общего вида (1) при условии четности.
} 
Третья, и последняя нужная нам группа

$$
K(\mathscr{D}(M)) /(K(\partial M) \oplus K(M))
$$

порождена классическими краевьми задачами $(P=1)$ и получается той же конструкцией, что и выше, только с заменой требования четности проектора $P$ на условие $P=1$. При этом получающаяся факторгруппа, аналогичная (33), на самом деле не требует новых обозначений, так как она может быть отождествлена с обычной $K$-группой векторных расслоений с компактньм носителем

$$
K(\mathscr{D}(M)) /(K(\partial M) \oplus K(M)) \stackrel{\gamma}{\longrightarrow} K_{c}\left(T^{*}(M \backslash \partial M)\right) \equiv K_{c}\left(T^{*} \stackrel{\circ}{M}\right) .
$$

Напомним конструкцию отображения $\gamma$. Во-первых, используем изоморфизмы

$$
K_{c}\left(T^{*}(M \backslash \partial M)\right) \simeq K\left(B^{*} M, \partial\left(B^{*} M\right)\right), \quad \partial\left(B^{*} M\right)=\left.S^{*} M \cup B^{*} M\right|_{\partial M},
$$

где $B^{*} M$ - расслоение единичных шаров многообразия $M$, а $\partial\left(B^{*} M\right)$ - полная граница этого расслоения. Это позволяет отождествлять Элементы групшы $K_{c}\left(T^{*} \stackrel{\circ}{M}\right)$ при помощи разностной конструкции как с изоморфизмами векторных расслоений $E, F \in \operatorname{Vect}(M)$

$$
\sigma: \pi^{*} E \rightarrow \pi^{*} F, \quad \pi: S^{*} M \rightarrow M,
$$

которые не зависят от $\xi$ над окрестностью края, так и с изоморфизмами, определенными всюду на $\partial\left(B^{*} M\right)$.

Рассмотрим классическую краевую задачу $(D, 1)$ вида $(2)$. Очевидно, что в окрестности края она может быть записана в виде задачи из примера 1. Тогда гомоморфизм $\gamma$ по определению равен

$$
\gamma(D, 1) \stackrel{\text { def }}{=} \sigma(D)(\xi)+i \chi(t)
$$

где $\chi(t)$ - срезающая функция, как и вьше, равная 1 на $\partial M$. Из формулы (24) следует, что этот символ (36) обратим на пространстве $\partial\left(B^{*} M\right)$. Нетрудно также построить и обратное к $\gamma$ отображение

$$
K_{c}\left(T^{*} \stackrel{\circ}{M}\right) \stackrel{\gamma^{\prime}}{\longrightarrow} K(\mathscr{D}(M)) /(K(\partial M) \oplus K(M)) .
$$

Изоморфизму $\sigma$ (см. (35)), который не зависит от $\xi$ над окрестностью края $\partial M$, это отображение сопоставляет классическую краевую задачу (2) следующим образом. По символу $\sigma$ строится эллиптический псевдодифференциальный оператор первого порядка в окрестности края, имеющий вид

$$
D=\sigma \circ \Lambda
$$

где $\sigma=\sigma(x)$ - гомоморфизм расслоений, а $\Lambda$ имеет главный символ $|\xi|$. Теперь остается только модифицировать оператор $D$ в окрестности края так, как это было уже сделано в примере 1:

$$
D^{\prime}=\sigma \circ\left((1-\chi(t)) \Lambda+\chi(t)\left(-\frac{\partial}{\partial t}+\Lambda_{+}\right)\right),
$$


где оператор $\Lambda_{+}$имеет главньй символ $\left|\xi^{\prime}\right|$. Наконец, определим

$$
\gamma^{\prime}(\sigma) \stackrel{\text { def }}{=}\left[D^{\prime}\right] \in K(\mathscr{D}(M)) /(K(\partial M) \oplus K(M))
$$

Это определение корректно, поскольку оператор $D^{\prime}$ задает фредгольмову краевую задачу без всяких краевых условий.

ЗАмЕчАниЕ 5. В терминах изоморфизма (37) легко доказать формулу индекса классических краевых задач вида (2)

$$
\operatorname{ind}(D, 1)=p_{!} \gamma(D, 1), \quad p: \stackrel{\circ}{M} \rightarrow p t,
$$

a $p_{!}: K_{c}\left(T^{*} \stackrel{\circ}{M}\right) \rightarrow K(p t)=\mathbb{Z}$ - отображение прямого образа в $K$-теории.

Действительно, рассмотрим краевую задачу $\left(D^{\prime}, 1\right)$ на многообразии $M$, которая совпадает с исходной $(D, 1)$ в окрестности края и является на всем многообразии задачей из примера 1. Рассмотрим композицию

$$
D_{0}=\left(D^{\prime}, 1\right)^{-1} \circ(D, 1)
$$

Главньй символ псевдодифференциального оператора $D_{0}$ в окрестности края является тождественным изоморфизмом расслоений. Поэтому для его индекса справедлива формула Атьи-Зингера

$$
\text { ind } D_{0}=p_{!}\left[\sigma\left(D_{0}\right)\right], \quad\left[\sigma\left(D_{0}\right)\right] \in K_{c}\left(T^{*} \stackrel{\circ}{M}\right)
$$

Но краевая задача $\left(D^{\prime}, 1\right)$ имеет нулевой индекс и определяет нулевой элемент в $K$-групше

$$
\gamma\left(D^{\prime}, 1\right)=0 \in K_{c}\left(T^{*} \stackrel{\circ}{M}\right)
$$

Поэтому получаем требуемое

$$
\operatorname{ind}(D, 1)=\operatorname{ind} D_{0}=p !\left[\sigma\left(D_{0}\right)\right]=p_{!} \gamma(D, 1)
$$

ЗАмечАниЕ 6. Редукции предыдушего параграфа задают изоморфизм группы (34) и групшы классов стабильно гомотопически эквивалентных классических краевых задач (1). Таким образом, рассмотрение спектральных задач (2) не является ограничением общности и покрывает общий случай.

Три введенные группы связаны следующей точной последовательностью.

ПРЕДЛОЖЕНИЕ 2. Последовательность

$$
K_{c}\left(T^{*} \stackrel{\circ}{M}\right) \stackrel{\alpha}{\longrightarrow} K\left(\mathscr{D}_{\mathrm{ev}}(M)\right) /(K(\partial M) \oplus K(M)) \stackrel{\beta}{\longrightarrow} K\left(P_{\mathrm{ev}}(\partial M)\right) / K(\partial M)
$$

является точной. Здесь $\alpha$ отвечает вложению классических задач в четнъе, а отображсение $\beta$ индуцировано отображением “забывания" $(D, P) \rightarrow P$. 
ДокаЗАтельство. Равенство $\beta$ о $\alpha=0$ очевидно, так как проектор в классической краевой задаче является единичньм и тем самым определяет нулевой элемент в группе $K\left(P_{\mathrm{ev}}(\partial M)\right) / K(\partial M)$.

Проверим теперь включение $\operatorname{ker} \beta \subset \operatorname{Im} \alpha$. Пусть для четной задачи $(D, P)$ имеет место равенство

$$
\beta[D, P]=0 \in K\left(P_{\mathrm{ev}}(X)\right) / K(X) .
$$

Это означает, что существует гомотопия четных проекторов, соединяющая проектор $P$ и проектор $P^{\prime}=P_{C}{ }^{\infty}\left(\partial M, G^{\prime}\right)$, на пространство сечений некоторого расслоения на $\partial M$. Эту гомотопию обозначим через $P_{t}: P_{0}=0, P_{1}=P^{\prime}$. Поднимем гомотопию проекторов до гомотопии спектральных задач $\left(D_{t}, P_{t}\right)$. Тогда в групе Гротендика $K\left(\mathscr{D}_{\mathrm{ev}}(M)\right) /(K(\partial M) \oplus K(M))$ имеют место равенства

$$
[D, P]=\left[D_{1}, P_{1}\right]=\alpha\left[D_{1}, 1_{C^{\infty}\left(\partial M, G^{\prime}\right)}\right]
$$

так как краевая задача $\left(D_{1}, P_{1}\right)$ является классической.

Точность последовательности (38) установлена.

Далее будем предполагать, что многообразие $M$ является четномерным. Третий член последовательности (38) в этом случае упрошается: согласно формуле (12) конечномерные проекторы порождают в этом члене подгруппу, изоморфную $\mathbb{Z}$ :

$$
\mathbb{Z} \subset K\left(P_{\text {ev }}(X)\right) / K(X),
$$

а факторгруппа

$$
K\left(P_{\mathrm{ev}}(X)\right) /(K(X) \oplus \mathbb{Z}) \simeq K\left(P^{*} X\right) / K(X), \quad P^{*} X-\text { проективизация } S^{*} X,
$$

согласно [8] состоит из элементов конечного порядка, который является степенью 2.

Аналогичньм образом в группе Гротендика четных краевых задач есть подгруппа

$$
\mathbb{Z} \subset K\left(\mathscr{D}_{\mathrm{ev}}(M)\right) /(K(\partial M) \oplus K(M)),
$$

порожденная краевыми задачами для нулевых операторов с конечномерными проекторами в правых частях. Это позволяет уточнить последовательность (38):

$$
K_{c}\left(T^{*} \stackrel{\circ}{M}\right) \stackrel{\alpha}{\longrightarrow} K\left(\mathscr{D}_{\mathrm{ev}}(M)\right) /(K(\partial M) \oplus K(M) \oplus \mathbb{Z}) \stackrel{\beta}{\longrightarrow} K\left(P^{*}(\partial M)\right) / K(\partial M) .
$$

Для четной краевой задачи определим аналог топологического индекса АтьиЗингера. В этом случае рассмотрим дубль многообразия $M$ :

$$
2 M \stackrel{\text { def }}{=} M \bigcup_{\partial M} M .
$$

На этом пространстве имеется (непрерывный) эллиптический символ

$$
\sigma(\widetilde{D})(\xi)=\sigma(D)(\xi) \cup \sigma(D)(-\xi)
$$

на первой копии дубля этот символ равен исходному $\sigma(D)(\xi)$, а на второй копии равняется $\sigma(D)(-\xi)$. Непрерьвность в месте склейки следует из равенства

$$
\sigma(D)\left(-\tau, \xi^{\prime}\right)=\sigma(D)\left(-\tau,-\xi^{\prime}\right)
$$

В этих обозначениях имеет место формула индекса четных краевых задач, устанавливаемая следующей теоремой. 
ТЕОРема 5. Имеет место формула индекса

$$
\operatorname{ind}(D, P)=\frac{1}{2} \operatorname{ind}_{t}(\sigma(\widetilde{D}))-d(P),
$$

где $d(P)$ - размерность проектора $P$ при нулевой нормировке (см. теорему 1$)$.

ДокАЗАТЕЛЬСтво. Индекс краевой задачи и правая часть формулы (40) продолжаются до гомоморфизмов абелевых групп, обозначаемых соответственно

$$
\text { ind, } \operatorname{ind}^{\prime}: K\left(\mathscr{D}_{\mathrm{ev}}(M)\right) /(K(\partial M) \oplus K(M)) \rightarrow \mathbb{Q} \text {. }
$$

Воспользуемся теперь точной последовательностью (39). Проверим сначала, что оба гомоморфизма совпадают на классических краевых задачах. В этом случае имеем $d(P)=0$, так как проектор единичный, а нормировка $\chi$ выбрана нулевой. Поэтому для классических краевых задач надо проверить выполнение формулы индекса

$$
\operatorname{ind}(D, 1)=\frac{1}{2} \operatorname{ind}_{t}(\sigma(D)(\xi) \cup \sigma(D)(-\xi)) .
$$

В силу изоморфизма (37) левая и правая части формулы (41) являются гомоморфизмами групп

$$
K_{c}\left(T^{*} \stackrel{\circ}{M}\right) \rightarrow \mathbb{Q} .
$$

Правая часть в формуле (41) для классических краевых задач раскладывается на два слагаемых

$$
\begin{gathered}
\operatorname{ind}_{t}(\sigma(D)(\xi) \cup \sigma(D)(-\xi))=\operatorname{ind}_{t}(\sigma(D)(\xi))+\operatorname{ind}_{t}(\sigma(D)(-\xi)), \\
\sigma(D)(\xi), \sigma(D)(-\xi) \in K_{c}\left(T^{*} \stackrel{\circ}{M}\right) .
\end{gathered}
$$

Покажем, что на четномерном многообразии оба слагаемых последней формулы равны. Действительно, в когомологической формулировке имеем

$$
\operatorname{ind}_{t}(\sigma(D)(-\xi))=\left\langle\operatorname{ch}[\sigma(D)(-\xi)] \operatorname{Td}\left(T^{*} M \otimes \mathbb{C}\right),\left[T^{*} \stackrel{\circ}{M}\right]\right\rangle \text {. }
$$

Инволюция $\xi \rightarrow-\xi$ пространства $T^{*} M$ сохраняет его ориентацию. Поэтому из (42) получаем

$$
\operatorname{ind}_{t}(\sigma(D)(\xi))=\operatorname{ind}_{t}(\sigma(D)(-\xi)) .
$$

Теперь равенство в (41) следует из формулы индекса классических краевых задач (см. замечание 5)

$$
\operatorname{ind}(D, 1)=\operatorname{ind}_{t}(\sigma(D)(\xi))
$$

Гомоморфизмы ind и ind ${ }^{\prime}$, очевидно, совпадают также и на подгруппе $\mathbb{Z}$, порожденной краевьми задачами для нулевых операторов. Таким образом, разность ind - ind' опускается до гомоморфизма факторгруппы

$$
\text { ind }-\operatorname{ind}^{\prime}:\left[K\left(\mathscr{D}_{\mathrm{ev}}(M)\right) /(K(\partial M) \oplus K(M) \oplus \mathbb{Z})\right] / \operatorname{Im} \alpha \rightarrow \mathbb{Q} \text {. }
$$


Из точности последовательности (39) следует, что $\operatorname{Im} \alpha=\operatorname{ker} \beta$. Заметим, что гомоморфизм $\beta$ принимает значения в группе кручения (см. последовательность (39)). Отсюда следует, что разность ind - ind' не может быть ненулевой, так как она определена на групте, состояшей из элементов конечного порядка.

Теорема 5 доказана.

Изученные выше операторы в подпространствах на замкнутых многообразиях и краевые задачи на многообразиях с границей являются взаимосвязанными.

Пусть $D$ - оператор на замкнутом многообразии $M$, действуюший в подпространствах

$$
D: \operatorname{Im} P_{1} \rightarrow \operatorname{Im} P_{2}, \quad \operatorname{Im} P_{1,2} \subset C^{\infty}\left(M, E_{1,2}\right) .
$$

Сопоставим ему некоторую краевую задачу.

На цилиндре $M \times[0,1]$ рассмотрим эллиптический оператор первого порядка с постоянными вдоль цилиндра коэффициентами

$$
D^{\prime}=\frac{\partial}{\partial t}+\left(1-P_{1}\right) \Lambda\left(1-P_{1}\right)-P_{1} \Lambda P_{1}: C^{\infty}\left(M \times[0,1], E_{1}\right) \rightarrow C^{\infty}\left(M \times[0,1], E_{1}\right)
$$

для положительного самосопряженного оператора первого порядка $\Lambda$ на многообразии $M$, имеющего главный символ, равный $|\xi|$. На двух компонентах края поставим краевые условия: на одной части спектральное, а на другой общее:

$$
\left\{\begin{array}{l}
\left(1-P_{1}\right) u(0)=g \in \operatorname{Im}\left(1-P_{1}\right) \subset C^{\infty}\left(M, E_{1}\right) \\
D u(1)=g^{\prime} \in \operatorname{Im} P_{2} \subset C^{\infty}\left(M, E_{2}\right)
\end{array}\right.
$$

Заметим, что эллиптичность краевых условий (45) эквивалентна эллиптичности оператора в подпространствах (43).

ПРЕДЛОЖЕНИЕ 3. Индекс краевой задачи (44), (45) равен индексу оператора в подпространствах (43).

ДокАЗАТЕЛЬство. Это утверждение достаточно доказать в случае, когдапроекторы $P_{1,2}$ являются ортогональными. В этой ситуации оператор (44) имеет вид

$$
D^{\prime}=\frac{\partial}{\partial t}+A, \quad A-\text { самосопряженный оператор, }
$$

что позволяет свести доказательство равенства индексов к непосредственной выкладке (с использованием собственных функций оператора $A$ ). Соответствующие вычисления мы для краткости опустим.

\section{§6. $\eta$-инварианты и четные проекторы}

Напомним [8], что классический псевдодифференциальньй оператор $A$ целого положительного порядка $m$ называется допустимыц, если его полный символ

$$
a(x, \xi) \sim a_{m}(x, \xi)+a_{m-1}(x, \xi)+\cdots
$$


удовлетворяет условиям четности

$$
a_{\alpha}(x,-\xi)=(-1)^{\alpha} a_{\alpha}(x, \xi) \quad \forall \xi \neq 0, x, \alpha=m, m-1, m-2, \ldots
$$

(допустимость оператора не зависит от выбора системы координат, в которой рассматривается полньй символ). Напомним также, что $\eta$-функиия самосопряженного оператора $A$ равна

$$
\eta(s, A)=\sum_{\lambda \in \operatorname{spec} A} \operatorname{sign} \lambda|\lambda|^{-s},
$$

где суммирование производится по собственным значениям оператора $A$ (с учетом кратности). Хорошо известно, что для эллиптических самосопряженных операторов на нечетномерных многообразиях $\eta$-инвариант Атьи-Патоди-Зингера [3], который по определению равен значению в нуле аналитического продолжения $\eta$-функции, является конечным числом, т.е. $\eta$-функция не имеет полюса в начале координат. Предполагая далее, что $A$ - допустимьй оператор четного порядка на нечетномерном многообразии, можно утверждать, что редукция $\eta$-инварианта по модулю $\mathbb{Z}$ инвариантна относительно деформаций оператора, а скачки появляются только в результате (разрывных) изменений неотрицательного спектрального подпространства оператора.

Такая гомотопическая инвариантность наводит на мысль, что в этом классе операторов $\eta$-инвариант оператора $A$ полностью определяется его неотрицательным собственным подпространством (это подпространство является, в действительности, образом четного проектора). Эта идея реализована в следующем утверждении.

ПРЕДЛОЖЕНИЕ 4. Пусть $P_{+}-$четный псевдодифференциальный проектор, равный неотрицательному спектральному проектору для некоторого допустимого оператора А. Тогда размерность проектора при нулевой нормировке $\chi: K(M) \rightarrow \mathbb{R}$ и $\eta$-инвариант оператора А равньи:

$$
d\left(P_{+}\right)=\frac{\eta(0, A)+\operatorname{dim} \operatorname{ker} A}{2} \stackrel{\text { def }}{=} \eta(A) .
$$

ДокАЗАТЕльство. Порядки элементов группы $K\left(P^{*} M\right) / K(M)$ являются степенями 2. Поэтому (см. $§ 1$ и работу [8]) для достаточно большого $N$ оператор $2^{N} A$ гомотопен (в пространстве допустимых самосопряженных эллиптических операторов) некоторому оператору $A^{\prime}$, который равен прямой сумме положительного и отрицательного допустимых операторов. Отсюда получаем, что $\eta\left(A^{\prime}\right)=0$ (см. [8]). Обозначим произвольную такую гомотопию через $A_{t}$. Вспоминая, что спектральньй поток семейства $A_{t}$ через точку нуль равен полному числу скачков $\eta$-инварианта, имеем

$$
\eta\left(2^{N} A\right)=-\operatorname{sf} A_{t}
$$

С другой стороны, рассматривая спектральное сечение (см. [10]) $P_{t}$ семейства $A_{t}$, можно показать (и это одно из определений спектрального потока), что

$$
\operatorname{sf} A_{t}=-\operatorname{ind}\left(2^{N} P_{+}, P_{0}\right)+\operatorname{ind}\left(P_{+, A^{\prime}}, P_{1}\right) .
$$


Последнее выражение равно $d$-размерности (см. замечание 1 )

$$
-\operatorname{ind}\left(2^{N} P_{+}, P_{0}\right)+\operatorname{ind}\left(P_{+, A^{\prime}}, P_{1}\right)=-d\left(2^{N} P_{+}\right)+d\left(P_{+, A^{\prime}}\right)=-d\left(2^{N} P_{+}\right) .
$$

Наконец, получаем требуемое

$$
\eta(A)=\frac{1}{2^{N}} \eta\left(2^{N} A\right)=\frac{1}{2^{N}} d\left(2^{N} P_{+}\right)=d\left(P_{+}\right) .
$$

ЗАмечание 7 . Четньй проектор $P$ удовлетворяет условиям предложения 4 тогда и только тогда, когда он сам является допустимым оператором нулевого порядка.

В самом деле, из допустимости оператора $A$ следует допустимость его спектрального проектора (см. [8]). Для доказательства обратного утверждения заметим, что допустимые операторы образуют алгебру. Рассмотрим теперь эллиптический оператор

$$
A=P \Delta P-(1-P) \Delta(1-P),
$$

где $\Delta$ обозначает произвольньй допустимый положительный самосопряженный оператор второго порядка с главньм символом лапласиана $|\xi|^{2}$. Оператор $A$ есть в точности требуемый (т.е. оператор с неотрицательным спектральным проектором, равным $P$ ). Последнее завершает доказательство замечания.

В цитированной работе [8] изучался вопрос о топологическом смысле инварианта $\eta \bmod \mathbb{Z}$ (т.е. по модулю скачков). В этом случае было замечено, что такая редукция корректно определяет гомоморфизм

$$
\eta: K\left(P^{*} M\right) / K(M) \rightarrow \mathbb{Z}\left[\frac{1}{2}\right] \bmod \mathbb{Z} .
$$

В силу предложения 4 полученная формула индекса операторов в подпространствах $(15)$, редуцированная $\bmod \mathbb{Z}$, дает новую формулу для $\eta$-инварианта (но не для всех элементов с главными символами из группы $\left.K\left(P^{*} M\right)\right)$, которую сформулируем в виде следствия.

\section{СЛЕДСТВИЕ 1. Пусть}

$$
W \in \operatorname{ker}\left\{\pi^{*}: K\left(P^{*} M\right) / K(M) \rightarrow K\left(S^{*} M\right) / K(M)\right\}, \quad \pi: S^{*} M \rightarrow P^{*} M .
$$

Тогда дробная часть $\eta$-инварианта расслоения $W$ полуцелочисленна и равна

$$
\begin{gathered}
\eta(W) \equiv \frac{1}{2} \operatorname{ind}_{t}\left(\sigma(\xi) \sigma^{-1}(-\xi): \pi_{S}^{*} F \rightarrow \pi_{S}^{*} F\right) \bmod \mathbb{Z}, \\
\pi_{S}: S^{*} M \rightarrow M, \quad F \in \operatorname{Vect}(M)
\end{gathered}
$$

а изоморфизм $\sigma$ - произвольный изоморфизм векторных расслоений $\pi^{*} W$ и $\pi_{S}^{*} F$ над расслоением кокасательных сфер, существующий в силу условия (46).

Формула индекса четных краевых задач приводит к аналогичной формуле (cp. [18]). 
СлЕДСтВИЕ 2. Пусть $(D, B, P)$ - четная әллиптическая краевая задача на многообразии $M$. Тогда дробная часть $\eta$-инварианта четного расслоения $\operatorname{Im} \sigma(P)$ является полуцелой и можст быть вычислена по формуле

$$
\eta(\operatorname{Im} \sigma(P)) \equiv \frac{1}{2} \operatorname{ind}_{t}(\sigma(D)(\xi) \cup \sigma(D)(-\xi)) \bmod \mathbb{Z}
$$

Из последнего утверждения получаем инвариантность относительно кобордизма, которую сформулируем в терминах $K$-теории. Перед этим напомним некоторые конструкции из работы [12].

Рассмотрим гомоморфизм

$$
\begin{gathered}
K\left(S^{*} X\right) / K(X) \rightarrow K_{c}\left(T^{*} X \oplus 1\right), \\
W=\operatorname{Im} P \subset \pi^{*} E \rightarrow[\tau+i(2 P-1)|\xi|],
\end{gathered}
$$

где $E \in \operatorname{Vect}(X), \pi: S^{*} X \rightarrow X-$ естественная проекция, а элемент

$$
[\tau+i(2 P-1)|\xi|] \in K_{c}\left(T^{*} X \oplus 1\right)
$$

понимается в смысле разностной конструкции, так как он вне нулевого сечения расслоения $T^{*} X \oplus 1$ задает изоморфизм в поднятии расслоения $E$ на пространстве $T^{*} X \oplus 1$. Известно [12], что этот гомоморфизм совпадает с композицией

$$
K\left(S^{*} X\right) / K(X) \stackrel{\delta}{\longrightarrow} K^{1}\left(T^{*} X\right)=K_{c}\left(T^{*} X \times \mathbb{R}\right),
$$

где $\delta$ индуцировано кограничным оператором в $K$-теории. Более того, отображение $\delta$ является изоморфизмом (для нечетномерного многообразия $X$ это легко проверить, воспользовавшись ненулевьм векторньм полем). Используя отождествление (49), задаваемое формулой (48), нетрудно получить утверждение об инвариантности относительно кобордизма в следующей форме.

СЛЕДСТВИЕ 3. Пусть на крае $X=\partial M$ гладкого четномерного многообразия $M$ задано четное расслоение $[W] \in K\left(P^{*} X\right) / K(X)$. Пусть его поднятие на расслоение кокасательных сфер

$$
\pi^{*}[W] \in K\left(S^{*} X\right) / K(X), \quad \pi: S^{*} X \rightarrow P^{*} X
$$

продолжается внутрь многообразия $M$, т.е. лежсит в образе оператора ограничения

$$
i^{*}: K_{c}\left(T^{*} M\right) \rightarrow K_{c}\left(\left.T^{*} M\right|_{X}\right),\left.\quad T^{*} M\right|_{X} \subset T^{*} M, \quad \pi^{*}[W] \in \operatorname{Im} i^{*}
$$

(при отожсдествлении (49)). Тогда удвоенный $\eta$-инвариант расслоения $W$ является иельм:

$$
2 \eta(W) \bmod \mathbb{Z}=0
$$




\section{§7. Примеры}

1. На компактном связном нечетномерном ориентированном многообразии $M$ рассмотрим эллиптический самосопряженньй дифференциальный оператор второго порядка, действующий на внешних 1-формах по формуле

$$
A=d \delta-\delta d: \Lambda^{1}(M) \rightarrow \Lambda^{1}(M),
$$

где $d$ - обычный оператор внешнего дифференцирования и $\delta$ - сопряженньй оператор. Прямое вычисление показывает, что главный символ неотрицательного спектрального проектора, обозначаемого через $P$, для этого оператора в произвольной точке $\xi \neq 0$ проектирует на прямую, порожденную самим ковектором $\xi$. Другими словами, проектор $P$ является четным проектором, а образ $\operatorname{Im} \sigma^{\prime}(P)$ его главного символа на каждом проективном пространстве $P_{x}^{*} M$ является в точности тавтологическим линейным расслоением, которое не является тривиальным. Вместе с тем поднятие этого расслоения на косферическое расслоение будет тривиальньм с естественной тривиализацией

$$
\kappa: \operatorname{Im} \sigma(P) \rightarrow \mathbb{C}, \quad \kappa(x, \xi, \eta)=(\xi, \eta)
$$

где $(\xi, \eta)$ - скалярное произведение двух пропорциональных векторов. Вычисления, приведенные в работе [8], показывают, что в этом случае

$$
\eta(A)=\left.\operatorname{dim} \operatorname{ker}(d \delta+\delta d)\right|_{\Lambda^{1}(M)}-1=\operatorname{dim} H^{1}(M)-1 .
$$

Поэтому для эллиптических операторов $D: \operatorname{Im} P \rightarrow C^{\infty}(M)$, действующих в соответствуюших подпространствах, формула индекса может быть переписана в виде

$$
\operatorname{ind}\left(D, P, P_{C^{\infty}(M)}\right)=\operatorname{ind}_{t}\left(\sigma(D) \kappa^{-1}: \pi^{*} \mathbb{C} \rightarrow \pi^{*} \mathbb{C}\right)+\operatorname{dim} H^{1}(M)-1 .
$$

Здесь $\operatorname{ind}_{t}\left(\sigma(D) \kappa^{-1}: \pi^{*} \mathbb{C} \rightarrow \pi^{*} \mathbb{C}\right)$ - топологический индекс обычного эллиптического псевдодифференциального оператора, действующего на функциях. ${ }^{7}$

2. Известен также аналог оператора $A$ из последнего примера для случая операторов с коэффициентами в расслоении $W \in \operatorname{Vect}(M)$, а также действующий на формах старших степеней. Именно, рассмотрим векторное расслоение $W$ со связностью $D$. Тогда соответствующий оператор определяется по формуле

$$
A \otimes 1_{W}=D D^{*}-D^{*} D: \Lambda^{k}(M, W) \rightarrow \Lambda^{k}(M, W) .
$$

Он также является эллиптическим и самосопряженньм. Его главный символ является тензорным произведением

$$
\sigma\left(A \otimes 1_{W}\right)=\sigma(A) \otimes 1_{W} .
$$

\footnotetext{
${ }^{7}$ При $\operatorname{dim} M \geqslant 3$ этот индекс, как хорошо известно, равен нулю. Для получения нетривиального индекса в этой ситуации можно рассматривать матричные операторы.
} 
В работе П. Гилки [8] была поставлена задача о вычислении дробной части $\eta$-инвариантов операторов такого вида. Из формулы (47) следует, например, что для таких операторов, действующих на 1-формах с коэффициентами в произвольном расслоении $W$, эта дробная часть равна нулю. Действительно, по формуле индекса она равна дробной части половины индекса псевдодифференциального оператора с постоянным главным символом.

Проблема, поставленная в цитированной работе П. Гилки, которая состоит в нахождении операторов с ненулевой дробной частью $\eta$-инварианта, остается открытой. В контексте рассматриваемых четных проекторов эта проблема может быть частично переформулирована следующим образом: возможно ли появление нецелых слагаемых в установленных нами формулах индекса?

\section{Список литературы}

1. Atiyah M.F., Bott R. The index problem for manifolds with boundary // Differ. Analysis, Bombay Colloquium. Oxford: Oxford Univ. Press, 1964. P. 175-186.

2. Стернин Б. Ю., Шаталов В.Е., Шульце Б.-В. Об общих краевых задачах для эллиптических уравнений // Матем. сб. 1998. Т. 189. № 10. С. 145-160.

3. Atiyah M. F., Patodi V. K., Singer I. M. Spectral asymmetry and Riemannian geometry. I // Math. Proc. Cambridge Philos. Soc. 1975. V. 77. P. 43-69.

4. Савин А. Ю., Стернин Б. Ю., Шульце Б.-В. Об инвариантных формулах индекса для спектральных краевых задач // Дифференц. уравнения. 1999. Т. 35. № 5. С. 705-714.

5. Савин А. Ю. Об операторах, допускающих разбиение формулы индекса спектральных краевых задач // Докл. РАН (в печати).

6. Hörmander L. The analysis of linear partial differential operators. III. Berlin: SpringerVerlag, 1985.

7. Назайкинский В.Е., Стернин Б. Ю., Шаталов В.Е., Шульце Б.-В. Спектральные краевые задачи и эллиптические уравнения на многообразиях с особенностями // Дифференц. уравнения. 1998. Т. 34. № 5. С. 695-708.

8. Gilkey P. B. The eta invariant of even order operators // Differential geometry. Proc. 3rd Int. Symp. (Peniscola, Spain, 1988). Lecture Notes in Math. V. 1410, 1989. P. 202-211.

9. Бирман М. С., Соломяк М. З. О подпространствах, допускающих псевдодифференциальный проектор // Вестн. ЛГУ. Сер. матем., мех., астроном. 1982. № 1. С. 18-25.

10. Melrose R. B., Piazza P. Families of Dirac operators, boundaries and the B-calculus // J. Differential Geom. 1997. V. 46. № 1. P. 99-180.

11. Wojciechowski $K$. A note on the space of pseudodifferential projections with the same principal symbol // J. Operator Theory. 1986. V. 15. № 2. P. 207-216.

12. Atiyah M.F., Patodi V.K., Singer I. M. Spectral asymmetry and Riemannian geometry. III // Math. Proc. Cambridge Philos. Soc. 1976. V. 79. P. 71-99.

13. Dai X., Zhang W. Splitting of the family index // Comm. Math. Phys. 1996. V. 182. № 2. P. 303-318.

14. Atiyah M.F., Bott R., Patodi V.K. On the heat equation and the index theorem // Invent. Math. 1973. V. 19. P. 279-330.

15. Atiyah M.F., Singer I. M. The index of elliptic operators. I // Ann. of Math. (2). 1968. V. 87. Р. 484-530; русский пер.: УМН. 1968. Т. 23. № 5. С. 99-142.

16. Rempel $S$., Schulze B.-W. Index theory of elliptic boundary problems. Berlin: AkademieVerlag, 1982.

17. Аәранович M. С., Вишик М. И. Эллиптические задачи с параметром и параболические задачи общего вида // УМН. 1964. Т. 19. №3. С. 53-161. 
18. Gilkey P. B. The eta invariant for even dimensional PIN $N_{c}$ manifolds // Adv. Math. 1985. V. 58. P. 243-284.

Московский государственнњй университет им. М.В. Ломоносова
Поступила в редакцию 30.10 .1998 